\title{
Impairment of acquisition of intravenous cocaine self-administration by RNA-interference of dopamine D1-receptors in the nucleus accumbens shell
}

\author{
Augusta Pisanu $^{\text {a, } 2}$, Daniele Lecca b, c, 2, Valentina Valentini ${ }^{\text {b, c, d }}$, Amine Bahi ${ }^{\text {e, }}$, \\ Jean-Luc Dreyer ${ }^{\mathrm{e}}$, Fabio Cacciapaglia ${ }^{\mathrm{f}}$, Andrea Scifo ${ }^{\mathrm{b}}$, Giovanna Piras ${ }^{\mathrm{b}, \mathrm{c}}$, \\ Cristina Cadoni ${ }^{\text {a, c, d }}$, Gaetano Di Chiara a, b, c, d, * \\ a Institute of Neuroscience, National Research Council of Italy, 09124 Cagliari, Italy \\ b Department of Biomedical Sciences, Neuropsychopharmacology Section, University of Cagliari, 09124 Cagliari, Italy

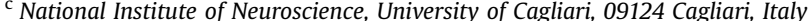 \\ d Centre of Excellence 'Neurobiology of Dependence', University of Cagliari, 09124 Cagliari, Italy \\ e Division of Biochemistry, Department of Medicine, University of Fribourg, CH-1700 Fribourg, Switzerland \\ ${ }^{\mathrm{f}}$ Department of Toxicology, University of Cagliari, 09124 Cagliari, Italy
}

\section{A R T I C L E I N F O}

\section{Article history:}

Received 25 July 2014

Received in revised form

20 October 2014

Accepted 21 October 2014

Available online 4 November 2014

\section{Keywords:}

D1a receptors

Dopamine

Nucleus accumbens shell

Cocaine

Self-administration

RNA-interference

\begin{abstract}
A B S T R A C T
Microdialysis during i.v. drug self-administration (SA) have implicated nucleus accumbens (NAc) shell DA in cocaine and heroin reinforcement. However, this correlative evidence has not been yet substantiated by experimental evidence obtained by studying the effect of selective manipulation of NAc shell DA transmission on cocaine and heroin SA. In order to investigate this issue, DA D1a receptor (D1aR) expression was impaired in the NAc shell and core by locally infusing lentiviral vectors (LV) expressing specific D1aR-siRNAs (LV-siRNAs). Control rats were infused in the same areas with LV expressing GFP. Fifteen days later, rats were trained to acquire i.v. cocaine or heroin self-administration (SA). At the end of behavioral experiments, in order to evaluate the effect of LV-siRNA on D1aR expression, rats were challenged with amphetamine and the brains were processed for immunohistochemical detection of cFos and D1aR. Control rats acquired i.v. cocaine and heroin SA. Infusion of LV-siRNAs in the medial NAc shell reduced D1aR density and the number of c-Fos positive nuclei in the NAc shell, while sparing the core, and prevented the acquisition of cocaine, but not heroin SA. In turn, LV-siRNAs infusion in the core reduced D1aR density and the number of c-Fos positive nuclei in the same area, while sparing the shell, and failed to affect acquisition of cocaine. The differential effect of LV impairment of NAc shell D1aR on cocaine and heroin SA indicates that NAc shell DA acting on D1aR specifically mediates cocaine reinforcement.
\end{abstract}

() 2014 Elsevier Ltd. All rights reserved.

\footnotetext{
Abbreviations: c-Fos-IR, c-Fos immunoreactivity; DA, dopamine; D1aR, D1a receptor; D1-IR, D1a R immunoreactivity; FR, fixed ratio; Lv-siRNA, lentivirus expressing siRNA; Lv-GFP, lentivirus expressing green fluorescent protein; NAc, nucleus accumbens; RNAi, RNA interference; SA, self-administration; siRNA, short interfering RNA; TH, tyrosine hydroxylase; TH-IR, TH immunoreactivity.

* Corresponding author. Department of Biomedical Sciences, Neuropsychopharmacology Section, University of Cagliari, Via Ospedale 72, 09124 Cagliari, Italy. Tel.: +39 0706758666.

E-mail address: gadichia@tiscali.it (G. Di Chiara).

1 Present address: Department of Psychology, College of Arts \&Sciences, University of Nebraska, Lincoln NE, USA.

2 Joint first authorship.
}

\section{Introduction}

A large body of evidence indicates that dopamine (DA) is the main neurochemical substrate of cocaine reinforcing properties. Early experimental studies by Ettenberg et al. (1982) showed that systemic administration of DA receptor antagonists elicits a compensatory increase of intravenous (i.v.) cocaine selfadministration (SA) in fully trained rats, thus mimicking the effect of non-reinforcement. In turn, discrete lesions of DA terminals by 6-OHDA point to the nucleus accumbens (NAc) as the DA projection area responsible for cocaine reinforcement (Pettit et al., 1984; Roberts et al., 1980). Studies on the effect of systemic and local intracerebral infusion of selective D1 antagonists (Caine et al., 
1995; Koob et al., 1987; Maldonado et al., 1993) on i.v. cocaine SA indicate a specific role of NAC DA D1 receptors.

Consistent with a role of NAc DA in cocaine behavioral effects, brain microdialysis studies have shown that response noncontingent administration of cocaine, amphetamine and most drugs of abuse, preferentially increases extracellular DA in the NAc, as compared to the dorsal caudate-putamen (Carboni et al., 1989; Di Chiara and Imperato, 1988). Microdialysis studies, in addition, have shown that cocaine increases extracellular DA in the NAc even after i.v. self-administration (Di Ciano et al., 1995; Pettit and Justice, 1989, 1991; Wise et al., 1995; Lecca et al., 2007a). Moreover, preferential stimulation of DA transmission in the ventral striatum by amphetamine has been demonstrated in humans by Positron Emission Tomography of $\left[{ }^{11} \mathrm{C}\right]$ raclopride binding (Drevets et al.,1999, 2001; Leyton et al., 2002; Martinez et al., 2003).

Anatomical studies have shown that the NAc is heterogenous with regards to its cytoarchitecture, connections and distribution of neurotransmitters, their enzymes and receptors. Thus, a medioventral part, the shell, and a dorso-lateral part, the core, have been distinguished (Groenewegen et al., 1999; Heimer et al., 1997). These two subdivisions, in turn, have been assigned different functions in behavior motivated by food and drugs of abuse (Corbit et al., 2001, Corbit and Balleine, 2011; Di Chiara, 2002; Di Chiara et al., 2004; Parkinson et al., 2002).

As far as DA transmission is concerned, cocaine, like other drugs of abuse, preferentially increases extracellular DA in the NAc shell as compared to the core (Di Chiara, 2002). This preferential action has been initially documented by microdialysis studies in acutely experimenter-administered rats (Aragona et al., 2008; Pontieri et al., 1995) and later demonstrated in rats during acquisition of i.v. cocaine SA (Lecca et al., 2007a).

The NAc shell, rather than the core, is also the area from which robust SA can be consistently obtained after response-contingent intracerebral infusion (Ikemoto, 2003; Rodd-Henricks et al., 2002), which, in turn, explains previous negative results obtained by infusions that did not specifically target the shell (Goeders and Smith, 1983).

The evidence presented above is suggestive, but, in order to demonstrate that NAc shell and core DA plays a differential role in cocaine reinforcement, a study of the effect of local impairment of DA transmission on i.v. cocaine SA is required. To our knowledge, four studies have directly compared the effect of impairment of DA transmission in the NAc shell and core on cocaine reinforcement. While in two studies no clear-cut differences were obtained between the two NAc subdivisions (Bachtell et al., 2005; Bari and Pierce, 2005), in the other two studies, the differences obtained have led to opposite conclusions (Di Ciano, 2008; Veeneman et al., 2012). These discrepancies and inconsistencies might result from the use of local intracerebral infusion of DA antagonists as a tool to manipulate DA transmission in the NAc shell and core. These drugs are highly lipophilic and rapidly diffuse out of their intracerebral target area, particularly after repeated administration. These difficulties call for the use of other means to impair DA transmission.

In recent years, RNA interference (RNAi), an evolutionary conserved mechanism of genome protection against viral infections and mutations (Hamilton and Baulcombe, 1999; Jensen et al., 1999; Sharp and Zamore, 2000), has been used to silence protein expression (Hannon and Rossi, 2004). RNAi is triggered in eukaryotic cells by double-stranded RNAs having 19-21 nucleotides (short interfering RNA, siRNA; Fire et al., 1998), which, when recognized as aberrant, induce a polyenzymatic process leading to degradation of homologous mRNAs (Hannon, 2002). For in vivo application, viral mediated expression of siRNAs, which takes place a few days after transduction (Bahi and Dreyer, 2012; Ortiz et al., 2010), allows stable long term RNAi, thus silencing the expression of the relative genes (Bahi et al., 2004, 2005; Bahi and Dreyer, 2012; Ortiz et al., 2010; Scherr et al., 2003; Van den Haute et al., 2003). Viral mediated silencing of targeted proteins offers higher temporal (RNAi starts right after transduction of targeted cells) and spatial control (inoculation of defined volumes and concentrations of viral vectors restricts silencing to targeted areas) of manipulations, compared to conventional knockout or pharmacological strategies. D1a (D1aR), rather than D1b receptors, are largely prevalent in striatal areas (Levey et al., 1993; Luedtke et al., 1999), including NAc (Muly et al., 2010). Therefore in the present study, D1aR silencing by lentiviral mediated expression of specific siRNA in discrete regions of rat NAc shell or core, was utilized to investigate the role of D1aR in cocaine reinforcement using an i.v. cocaine SA paradigm.

In order to test the specificity of the effect of D1aR silencing on the acquisition of cocaine reinforcement, the effect of the same manipulation on the acquisition of i.v. heroin SA was investigated. Heroin also increases dialysate DA preferentially in the NAc shell (Lecca et al., 2007b). Moreover, systemic flupentixol, a DA D2/D1 receptor antagonist, differentially affects responding for i.v. cocaine and heroin SA. At low doses it selectively increases responding for cocaine without affecting responding for heroin, while at higher doses, it reduces responding for both drugs (Ettenberg et al., 1982). These observations have been interpreted to mean that low doses of flupentixol selectively impair cocaine reinforcement, while higher doses exert a general effect on motivation and on its expression into action (Ettenberg et al., 1982). Given these premises, manipulation of DA D1aR expression by RNAi in the NAc shell versus core was utilized to investigate the role of DA in these two NAc subdivisions in cocaine reinforcement.

\section{Materials and methods}

2.1. Animals

Male Sprague-Dawley rats (Harlan, Italy), weighing 250-275 g at the beginning of experimental procedures, were housed four per cage with ad libitum food and water, under constant light-dark cycle (on 08:00 A.M., off 08:00 P.M.), temperature $\left(22{ }^{\circ} \mathrm{C}\right)$ and humidity (60\%). After catheter implantation and lentiviral stereotaxic injections, rats were individually housed in plastic cages $(30 \times 20 \times 20 \mathrm{~cm})$ in the same environmental conditions. During recovery (two weeks), rats were handled, treated with $0.1 \mathrm{ml}$ of gentamicin $(40 \mathrm{mg} / \mathrm{ml}$, i.v.), and their catheters were daily flushed with heparinized saline (heparin $25 \mathrm{IU}$ in $0.9 \%$ sterile saline).

Self-administration (SA) sessions were performed after recovery, during the light phase, between 10:00 A.M. and 5:00 P.M. and, after each experimental session, rats were returned to their home cages, where a daily ration of $20 \mathrm{~g}$ of food was made available.

All experimental procedures met the guidelines and protocols approved by the European Parliament and the Council of the European Union (directive 2010/63/EU, L 276/33 20/10/2010) and approved by the Ethical Committee for Animal Use and Care of the University of Cagliari. All efforts have been made to minimize suffering and the numbers of animals used.

\subsection{Drugs}

Cocaine hydrochloride (McFarlan Smith, Edinburgh, Scotland, UK) and heroin hydrochloride (NIDA, Research Triangle Park, NC, USA) were dissolved daily in sterile saline $(0.9 \%)$, and drug concentration was adjusted daily according to the weight of the rats. Amphetamine sulfate (Sigma, Milan, Italy) was dissolved in sterile saline $(0.9 \%)$ and administered subcutaneously. All reagents were obtained from local suppliers and were of analytical grade.

\subsection{Construction and validation of LV-Drd1a-siRNAs}

The construction of Drd1a-siRNA expressing lentiviruses has been described previously in detail (Bahi and Dreyer, 2012; Ortiz et al., 2010). In brief, three 19nucleotide Drd1a-siRNA sequences were added to the U6 promoter by PCR. The PCR product was digested with BamHI/Xhol and then ligated into the pTK431 previously digested with the same enzymes. Preparation of lentiviral vectors was initiated by triple transfection of HEK293T cells by calcium phosphate method, using pTK431 together with pDeltaNRF and pMDG-VSV. Cells were harvested $72 \mathrm{~h}$ later and viruses were concentrated from the supernatant by ultracentrifugation. Vectors were resuspended in PBS-BSA and stored at $-80^{\circ} \mathrm{C}$ until use (Bahi, 2013; Bahi and Dreyer, 2013). The three distinct Drd1a-siRNA sequences were successfully used to knock-down the expression Drd1a mRNA in the NAc (Bahi and Dreyer, 2012) and proteins in the hippocampus (Ortiz et al., 2010). 


\subsection{Catheter implant and lentivirus inoculation}

Rats were anaesthetized with Equitesin (0.97 g pentobarbital, $4.25 \mathrm{~g}$ chloral hydrate, $2.1 \mathrm{~g} \mathrm{MgSO}_{4}, 42.8 \mathrm{ml}$ propylene glycol, $11.5 \mathrm{ml} 90 \%$ ethanol $/ 100 \mathrm{ml} ; 5 \mathrm{ml} / \mathrm{kg}$ i.p.) and implanted into the right jugular vein with a catheter (Medical-grade tubing; Silastic, Dow Corning Corporation, Midland, MI, USA) fixed in the middle scapular region by a polypropylene mesh (Evolution, BULEV, weight $48 \mathrm{~g} / \mathrm{mq}$, Dipromed, Italy). This ensured stable fixation, rapid tissue integration and reduced foreign body reaction. During the same surgical procedure, rats underwent bilateral stereotaxic inoculation of lentiviral vectors in the NAc shell or core compartments; LV-Drd1asiRNA1, LV-Drd1a-siRNA2 and LV-Drd1a-siRNA3 concentrate stocks were mixed right before inoculation, and the LV-siRNAs mix and LV-GFP vectors were kept in ice at $0{ }^{\circ} \mathrm{C}$ until use. Stereotaxic inoculation was made using a 33-gauge tip needle connected by a polyethylene tubing to a $10-\mu \mathrm{L}$ Hamilton syringe, driven by an infusion pump (Basile, Milan, Italy).

For cocaine SA, lentiviral vectors were inoculated using two different experimental protocols (Experiment I and Experiment II). For Experiment I, $4 \mu \mathrm{l}$ of vector solution (LV-siRNAs or LV-GFP vectors) were bilaterally inoculated by a single injection in the NAc shell or core of 40 rats, using the following coordinates: i) NAc shell: $\mathrm{AP}+1.7 \mathrm{~mm}$; $\mathrm{L} \pm 1.1 \mathrm{~mm}$, from bregma; $\mathrm{V}-7.7 \mathrm{~mm}$ from dura, in LV-siRNAs shell and LV-GFP shell groups; ii) NAc core: A $+1.5 \mathrm{~mm}$; $\mathrm{L} \pm 1.8 \mathrm{~mm}, \mathrm{~V}-7.0 \mathrm{~mm}$, in LV-siRNAs core and LV-GFP core groups, according to the Paxinos and Watson Atlas (2005). For Experiment II, 28 rats were bilaterally inoculated in two different sites in the NAc shell or core compartments (rostro-caudal interval of $500 \mu \mathrm{m}$ ), and for each inoculation the volume was $2 \mu \mathrm{l}$. The following coordinates were used: i) NAc shell, caudal inoculation: A $+1.7 ; \mathrm{L} \pm 1,1 ; \mathrm{V}-7.7 \mathrm{~mm}$; rostral inoculation, $\mathrm{A}+2.2$; $\mathrm{L} \pm 0.8$; V $-7.2 \mathrm{~mm}$, for LV-siRNAs shell and LV-GFP shell groups; ii) NAc core, caudal inoculation: $\mathrm{A}+1.5 ; \mathrm{L} \pm 1.8 ; \mathrm{V}-7.0 \mathrm{~mm}$; rostral inoculation: $\mathrm{A}+2.0 ; \mathrm{L} \pm 1.6$; $\mathrm{V}-6.4 \mathrm{~mm}$, for LV-siRNAs core and LV-GFP core groups.

For heroin SA (Exp. III), $4 \mu \mathrm{l}$ of vector solution (LV-siRNAs or LV-GFP vectors) were bilaterally inoculated by a single injection in the NAc shell of 12 rats/group using the following coordinates: $\mathrm{AP}+1.7 \mathrm{~mm} ; \mathrm{L} \pm 1.1 \mathrm{~mm}$, measured from bregma; $\mathrm{V}-7.7 \mathrm{~mm}$ from dura, in LV-siRNAs shell and LV-GFP shell groups, according to the Paxinos and Watson Atlas (2005).

The rate of infusion was $0.2 \mu \mathrm{l} / \mathrm{min}$; the cannula was maintained in place for further 10 min after inoculation and then gradually withdrawn in order to allow a proper vector diffusion into the injected brain areas and to prevent their backflow through the needle track.

\subsection{Cocaine self-administration}

After 15 days of recovery from surgical procedures, which is sufficient to reach a maximal degree of D1aR silencing in vivo (Ortiz et al., 2010), rats were trained to self-administer cocaine intravenously (unit doses, $0.25 \mathrm{mg} / \mathrm{kg}$ in $24 \mu \mathrm{l}$ ) in single daily 1-h sessions for 5 days/week, under fixed ratio schedule 1 (FR-1, 1 nose-poke for 1 infusion), which was then increased to FR-5 (5 nose-pokes for 1 infusion) starting from the 15th session in each experiment. At this point, in at least one experimental group, rats reached the criterion for acquisition (set at $85 \%$ of responses on the active hole), keeping a stable pattern over three sessions. Starting from the 23rd (Experiment I) or the 34th session (Experiment II), rats were switched to extinction by substituting the cocaine solution with saline (Fig. 1). Each SA session was carried out in chambers $(31 \times 26 \times 33 \mathrm{~cm})$ housed in sound proof boxes (Coulbourn Instruments, Allentown, NJ, USA) and provided with two nose-poke holes, one active and the other one inactive, placed in the short walls of the cage $2 \mathrm{~cm}$ from the floor. A yellow/green light was placed over the active hole, and a red light over the inactive one, as discriminative stimuli. Prior to each daily session, the jugular catheter was flushed with $0.1 \mathrm{ml}$ of sterile saline, and rats were connected to the infusion system and placed into the Skinner box.

A computerized program (Graphic State 2 software, Coulbourn instrument, PA, USA) controlled each phase of SA session, with: 1) ready state, involving activation of the pump for $2 \mathrm{~s}$ to fill up the jugular catheter with cocaine solution (dead volume, $50-\mu \mathrm{l}) ; 2$ ) cocaine available state, involving FR-1 or FR-5 nose poking in the active hole; 3 ) infusion state, resulting in 2-s i.v. cocaine injection $(0.25 \mathrm{mg} / \mathrm{kg}$ in $24-\mu \mathrm{l}) ; 4)$ 20-s time-out state, during which further nose-pokes were recorded, but did not result in additional infusions. During the time-out, a scheduled house light was turned on, and both active and inactive holes were red lighted. After the time-out cocaine availability was once again indicated by turning on the yellow/green light in the active hole. Inactive nose-pokes were also recorded. At the end of the session, all light stimuli were turned off, the rats' catheter was flushed with $0.1 \mathrm{ml}$ of heparinized saline, and the animals were returned to their home cages. During the extinction phase, cocaine solution was replaced with saline, so that FR-5 active nosepoking during each SA session resulted in i.v. saline injections, under the same test conditions of previous cocaine SA sessions.

\subsection{Heroin self-administration}

In Exp. III, after recovery (15 days), rats were trained to i.v. heroin $(0.05 \mathrm{mg} / \mathrm{kg}$, $48 \mu \mathrm{l} / 4 \mathrm{~s}$ ) SA in single daily 1 -h sessions for 5 days/week under FR-1 schedule, which was then increased to FR-5 on the 11 th session, once rats had reached the criterion for acquisition. Starting from the 26th session, rats underwent extinction and heroin solution was substituted by saline (Fig. 1). Each heroin SA session was carried out in the same chambers and environmental conditions described for cocaine. During extinction, heroin solution was replaced with saline, so that FR-5 active nose-poking resulted in i.v. saline injections $(48 \mu \mathrm{l} / 4 \mathrm{~s})$.

\subsection{Immunohistochemistry}

In all experiments, the day after the last SA session, rats were challenged with saline or amphetamine ( $5 \mathrm{mg} / \mathrm{kg}$, s.c.). Two hours after challenge rats were deeply anaesthetized with Equitesin ( $5 \mathrm{ml} / \mathrm{kg}$, i.p.) and transcardially perfused with $100 \mathrm{ml}$ of ice-cold saline, followed by $250 \mathrm{ml}$ of ice-cold $4 \%$ paraformaldehyde (PFA) dissolved in $0.1 \mathrm{M}$ sodium phosphate buffer, $\mathrm{pH}$ 7.4.

\section{Experiment I

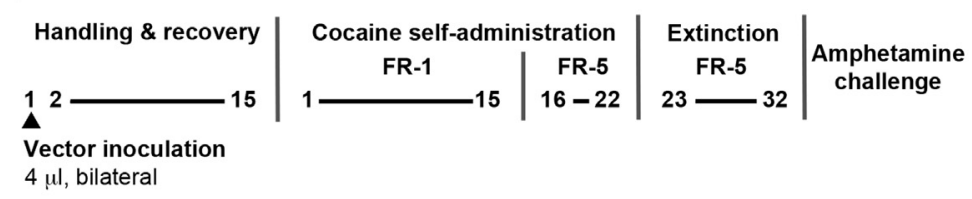

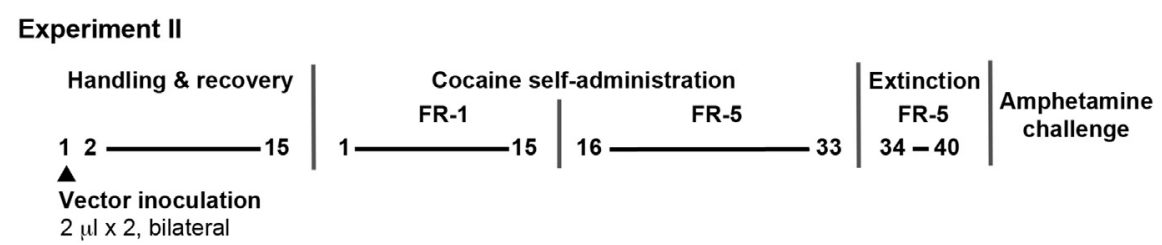

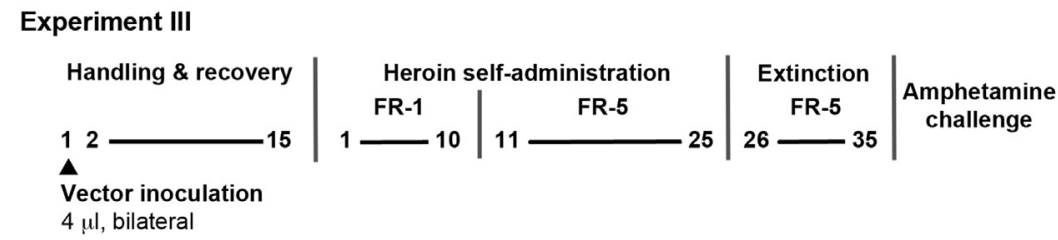

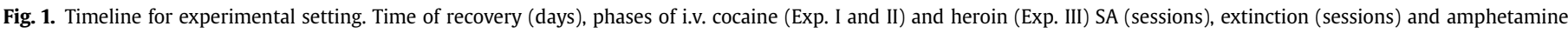
challenge are shown. Arrowheads indicate lentivirus inoculations. 
Brains were removed and post-fixed overnight with $4 \%$ PFA at $4{ }^{\circ} \mathrm{C}$. Serial coronal sections, which included NAc, were cut on a Vibratome $(40-\mu \mathrm{m}$ thickness: VT1000 S, Leica Microsystems Germany). Six sets of 6 sections, separated by a minimum of $240 \mu \mathrm{m}$, were collected for each animal so that adjacent sets of equivalent anteroposterior level could be used for immunostaining of D1aR, c-Fos protein and tyrosine hydroxylase $(\mathrm{TH})$ and for qualitative analysis of GFP expression.

Free floating sections were incubated with the primary antibodies (D1aR 1:1000, monoclonal anti-D1aR IgG raised in mouse; c-Fos, 1:2000, polyclonal anti-cFos IgG raised in sheep; Millipore, USA; TH, 1:1000, monoclonal anti-TH IgG raised in mouse, Sigma Aldrich, Italy). The reaction was visualized using biotinylated secondary antisera (1:500, donkey anti-mouse IgG biotin-SP-conjugate; $1: 700$, donkey anti-sheep IgG biotin-SP-conjugate, Jackson ImmunoResearch, UK) and standard avidin-biotin-horseradish peroxidase technique (ABC-élite kit, Vector Labs, UK, and Fast-DAB solution, Sigma-Aldrich, Italy). After this, sections were mounted on positively charged microscope slides, dehydrated, coverslipped using Eukitt mounting medium (Fluka, Sigma-Aldrich, Italy) and allowed to dry overnight.

Images were acquired with a Zeiss AxioScopeA1 microscope equipped with $20 \times / 0.45$ and $40 \times / 0.65$ Zeiss $\mathrm{N}$-Acroplan objectives, and a high resolution scanner for medical slides (Super Coolscan 9000ED, Nikon) set at $4000 \mathrm{dpi}$.

D1aR immunoreactivity (D1-IR) in the target area (NAc core and/or shell) was quantified as optical density, corrected for cortex signal meant as background, in images acquired by the high resolution scanner. D1-IR in the region of interest (ROI, inoculated area) was then expressed as percentage of the overall optical density of the related target area for each section analyzed. In adjacent sections, c-Fos immunoreactivity (c-Fos-IR) was quantified as counts/field of c-Fos positive nuclei in the ROI. Number of c-Fos positive nuclei was determined in the inoculated region of the NAc core and hell, in images acquired using $20 \times$ objective, by applying the "entropy threshold" and "analyze-particles" tools of Image J software (U.S. National Institutes of Health, USA) in a counting field of $500-\mu$ m diameter.

GFP autofluorescence was directly detected in a set of six sections for each rat inoculated with LV-GFP. Sections were rinsed in Trisma- $\mathrm{HCl}$ buffered solution ( $50 \mathrm{mM}, \mathrm{pH} 7.4$ ), mounted on positively charged microscope slides and coverslipped with Vectashield mounting medium (Vector Labs, UK). Images obtained with an epifluorescence microscope (Axio Scope. A1, Zeiss, Germany) using $20 \times$ and $40 \times$ objectives were acquired with a digital camera (1.4 MPixels, Infinity 3-1, Lumenera Canada).

\subsection{Data analysis}

Statistical analysis was carried out by Statistica 8 (Stat Soft Inc, Tulsa, OK, US).

\subsubsection{Self-administration}

A total of 8 rats each were excluded from Experiment I and Experiment Il respectively, due to catheter leakage during cocaine SA or incorrect intracerebra virus infusion, as indicated by immunohistochemical analysis. For the same reasons, 5 rats were excluded from Experiment III. The data recorded from these animals were excluded from analysis. Therefore the size of the analyzed and plotted data was derived from 8 rats for each group in Experiment I, 5 for Experiment II and 9-10 for Experiment III.

Nose poking behavior during each daily 1-h cocaine SA session and during extinction was analyzed by four-way ANOVA, with group (LV-siRNAs vs LV-GFPcontrols), area (NAc shell vs core) and cumulative nose-pokes (active $v s$ inactive) as between-subject factors, and session as repeated measure.

Cocaine intake and number of infusions were analyzed by three-way ANOVA with group (LV-siRNAs vs LV-GFP-controls) and area (NAc shell vs core) as betweensubject factors, and time as repeated measure of i) daily infusions and ii) weekly cocaine intake.

Nose poking behavior during each daily 1 -h heroin SA sessions and extinction was analyzed by three-way ANOVA, with group (LV-siRNAs vs LV-GFP-controls) and cumulative nose-pokes (active $v s$ inactive) as between-subject factors, and session as repeated measure.

Heroin intake and number of infusions were analyzed by two-way ANOVA, with group (LV-siRNAs vs LV-GFP-controls) as between-subject factors, and with time as repeated measure of $\mathrm{i}$ ) daily infusions and ii) weekly heroin intake.

If significant effects or interactions were obtained with ANOVA, multiple pai wise contrasts were made by Tukey's HSD or Fisher's LSD post-hoc test, as reported in each experiment. Significance was set at $P<0.05$.

\subsubsection{Immunohistochemistry}

D1-IR, quantified as percent (\%) of overall optical density of the target area, was analyzed by a three-way ANOVA, with group (LV-siRNAs vs LV-GFP), target area (NAc shell $v s$ core) and ROI (NAc shell $v s$ core) as factors in Experiment I and II, and by oneway ANOVA with group (LV-siRNAs vs LV-GFP) factor in Experiment III. c-Fos-IR quantified as counts/field of c-Fos-positive nuclei in the ROI, was analyzed by a fourway ANOVA with group (LV-siRNAs vs LV-GFP), target area (NAc shell vs core), ROI (NAc shell $v s$ core) and challenge (saline vs amphetamine, $5 \mathrm{mg} / \mathrm{kg}$ ) as betweensubject factors in Experiment I, three-way ANOVA, with group (LV-siRNAs vs LVGFP), target area (NAc shell vs core), ROI (NAc shell vs core) as between-subject factors in Experiment II, and one-way ANOVA with group (LV-siRNAs vs LV-GFP) as between subject factors in Experiment III.

If significant effects or interaction were found with ANOVA, Tukey's HSD posthoc test was then applied with significance set at $P<0.05$.

\section{Results}

\subsection{Experiment I}

\subsubsection{Cocaine self-administration}

In Experiment I, the effect of bilateral infusion of 4- $\mu$ l lentiviral vectors in the NAc shell (LV-siRNAs shell and LV-GFP shell groups) or core (LV-siRNAs core and LV-GFP core groups) on intravenous (i.v.) cocaine SA was evaluated. Rats were trained to acquire 1-h daily i.v. cocaine SA (unit dose $0.25 \mathrm{mg} / \mathrm{kg}, 5$ day/week) for 15 sessions under FR- 1 and for 7 sessions under FR- 5 schedules of responding, followed by 10 sessions of extinction (saline, $24 \mu \mathrm{l} /$ infusion).

3.1.1.1. Responding. Fig. 2 shows cumulative active and inactive nose-pokes performed by the LV-GFP and LV-siRNAs shell groups on panel A, and the LV-GFP and LV-siRNAs core groups on panel B, throughout the cocaine SA (FR-1: 1st-15th sessions, FR-5: 16th-22nd sessions) and extinction (FR-5: 23rd-32nd sessions) phases.

Four-way ANOVA with nose-pokes (active and inactive), group (LV-GFP and LV-siRNAs), area (shell and core) and session (days) as factors, showed a main effect of nose-pokes (active vs inactive; $\left.F_{1,56}=96.01, P=0.000001\right)$ and session $\left(F_{31,1736}=35.19\right.$, $P=0.000001)$ and significant group $\times \operatorname{session}\left(F_{31,1736}=2.13\right.$, $P=0.0003)$, nose-pokes $\times$ session $\left(F_{31,1736}=32.43, P=0.000001\right)$, group $\times$ area $\times$ session $\left(F_{31,1736}=2.94, P=0.00001\right)$ and group $\times$ area $\times$ nose poking $\times$ session $\left(F_{31,1736}=2.30, P=0.00007\right)$ interactions. The LV-GFP shell rats acquired cocaine SA behavior under FR-1 schedule during the second week and further increased active nose poking under FR-5 schedule $(P<0.05$ for active nose poking compared to inactive, Tukey's HSD post-hoc test), whereas the LV-siRNAs shell rats showed a delayed acquisition under FR-1 schedule, and lower active nose poking under FR-5 schedule, as compared to the LV-GFP shell controls (higher LV-GFP shell active nose poking compared to LV-siRNAs shell from the 16th to the 22nd session, $P<0.05$ Tukey's HSD post-hoc test). During extinction, substitution of cocaine with saline resulted in a marked fall of responding on the active hole during the first saline session itself in the LV-GFP shell rats, while the LV-siRNAs shell rats exhibited a pattern not different from previous FR-5 SA sessions, with only a slight reduction in nose poking during the last days $(P<0.05$ for active nose-poking compared to inactive, Tukey's HSD post-hoc test) (Fig. 2A).

Infusion of LV-siRNAs in the NAc core did not modify cocaine SA (Fig. 2B); active nose-pokes performed by the LV-siRNAs core rats were similar to those performed by the LV-GFP core control rats. Both the LV-GFP core and LV-siRNAs core groups rapidly acquired cocaine SA under FR-1 schedule during the 2nd week, and significantly increased their nose poking activity under FR-5 schedule (higher active nose-poking compared to inactive, $P<0.05$ Tukey's HSD post-hoc test). Moreover as seen for LV-GFP shell group, during the extinction phase both core groups quickly reduced their active nose poking behavior (Fig. 2B).

3.1.1.2. Cocaine intake. Three-way ANOVA, with group, area and session as factors, was applied to daily infusions of cocaine or saline. It showed a main effect of session $\left(F_{31,868}=26.72\right.$, $P=0.000001)$ and a group $\times \operatorname{session}\left(F_{31,868}=2.48, P=0.000017\right)$ and group $\times$ area $\times$ session $\left(F_{31,868}=1.85, P=0.003\right)$ interactions. 

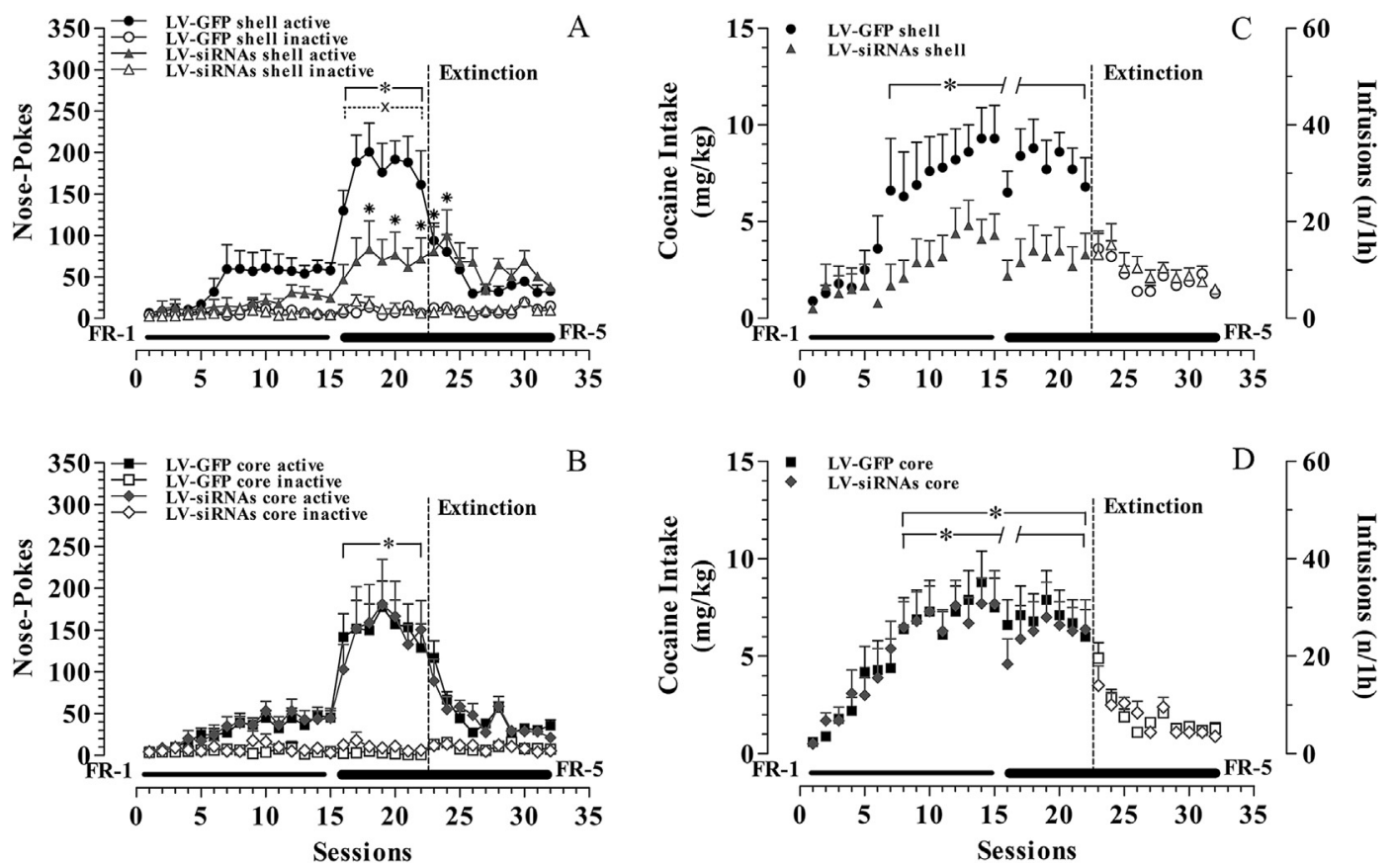

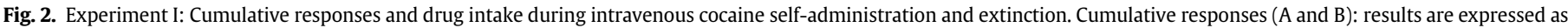

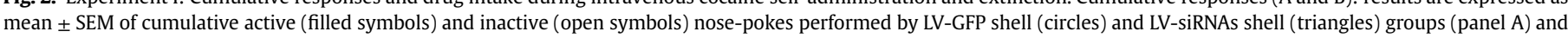

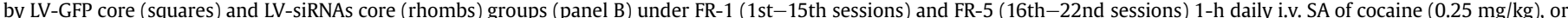

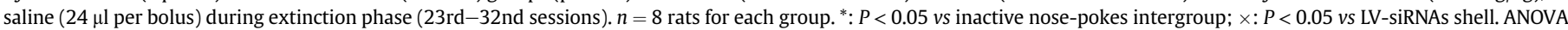

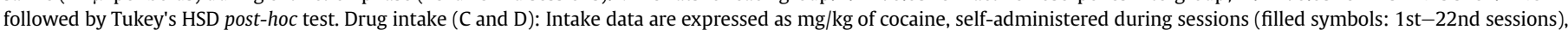

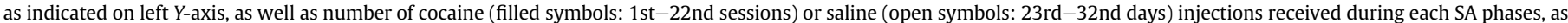

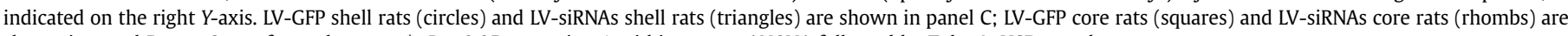
shown in panel $\mathrm{D}, n=8$ rats for each group. ${ }^{*}: P<0.05$ vs session 1 within group; ANOVA followed by Tukey's HSD post-hoc test.

As shown in Fig. 2C, the LV-GFP shell rats rapidly increased infusions of cocaine over days during the 2 nd week $(P<0.05$ vs 1 st day, Tukey's HSD post-hoc test), while the LV-siRNAs shell rats did not show any increase. On the other hand, both the LV-siRNAs core and LV-GFP core groups showed an increase starting from the 2nd week $(P<0.05$ vs 1 st day, Tukey's HSD post-hoc test. Fig. 2D). The number of cocaine infusions during the 16th session was affected by switching operant ratio schedule from FR-1 to FR-5.

Three-way ANOVA applied to cocaine intake, expressed as weekly means under FR-1 and FR-5 schedules of responding, showed similar results, with main effect for week $\left(F_{3,84}=36.24\right.$, $P=0.000001$ ), characterized by increase of weekly cocaine intake starting from the 2nd week in all experimental groups, except for the LV-siRNAs shell group $(P<0.05$ vs 1 st week, Tukey's HSD posthoc test, Table 1).

\subsubsection{Immunohistochemistry}

3.1.2.1. GFP expression. In vivo transfection was evaluated by green fluorescent protein (GFP) expression, detected in a set of six sections in the LV-GFP rats. In Experiment I, $4 \mu \mathrm{l}$ of LV-GFP, infused either in the NAc shell or core, spread $\approx 350 \mu \mathrm{m}$ rostrally and caudally from the inoculation site. Fig. 3 shows the sites of infusion (panels A and B) and GFP expression in the NAc shell and core after infusion of $4-\mu$ l Lenti-GFP (panels $E$ and $F$ ).

3.1.2.2. $T H$ expression. Integrity of presynaptic dopaminergic fibers in the NAc core and shell of silenced subjects was evaluated by immunohistochemical staining of TH. In fact, immunoreactivity for TH was not modified in the inoculated areas as indicated in Fig. 3 (panels A1-A4).
3.1.2.3. D1aR density and c-Fos expression. D1aR expression was quantified as percentage change of ROI optical density, compared to the overall optical density of the corresponding target area, while as an indirect measure of D1aR activation in virus infused regions, basal and amphetamine-induced c-Fos expression was quantified as counts/field of c-Fos positive nuclei.

D1aR and c-Fos immunoreactivity in the NAc shell and core of representative rats from Experiment I are shown in Fig. 4. Column B shows D1-IR, column C represents the pattern of nuclear c-Fos-IR distribution, while columns D and E are $20 \times$ magnifications of nuclear c-Fos-IR of the selected rectangular regions indicated in column C, relative to dorsal core and medial shell respectively.

As illustrated in column D of Fig. 4 (lines 1 and 2), c-Fos expression in the core of the LV-siRNAs shell rats was similar to that

Table 1

Experiment I. Mean + SEM of weekly cocaine intake per session $(\mathrm{mg} / \mathrm{kg})$ in LV-GFP and LV-siRNAs shell and core groups under FR-1 (1st-3rd week) and FR-5 (4th week) i.v. cocaine SA. $n=8$ rats per group.*: $P<0.05$ vs 1 st week; $\times: P<0.05$ vs respective GFP control group. ANOVA followed by Tukey's HSD post-hoc test.

\begin{tabular}{cllll}
\hline SA phases: & Groups: & & \\
\cline { 2 - 5 } & $\begin{array}{l}\text { LV-GFP } \\
\text { shell }\end{array}$ & $\begin{array}{l}\text { LV-siRNAs } \\
\text { shell }\end{array}$ & $\begin{array}{l}\text { LV-GFP } \\
\text { core }\end{array}$ & $\begin{array}{l}\text { LV-siRNAs } \\
\text { core }\end{array}$ \\
\hline FR-1 & & & & \\
1st week & $1.6 \pm 0.6$ & $1.3 \pm 0.8$ & $1.9 \pm 0.5$ & $2.0 \pm 0.5$ \\
2nd week & $6.2 \pm 2.1^{*}$ & $2.3 \pm 1.0^{\times}$ & $5.8 \pm 1.4^{*}$ & $6.0 \pm 1.3^{*}$ \\
3rd week & $8.6 \pm 1.5^{*}$ & $4.2 \pm 1.1^{\times}$ & $7.5 \pm 1.3^{*}$ & $7.2 \pm 1.3^{*}$ \\
FR-5 & & & & \\
4th week & $7.8 \pm 1.2^{*}$ & $3.1 \pm 1.1^{\times}$ & $6.9 \pm 1.3^{*}$ & $6.2 \pm 1.2^{*}$ \\
\hline
\end{tabular}

*: $P<0.05$ vs 1 st week

$\times: P<0.05$ vs respective GFP control group. 


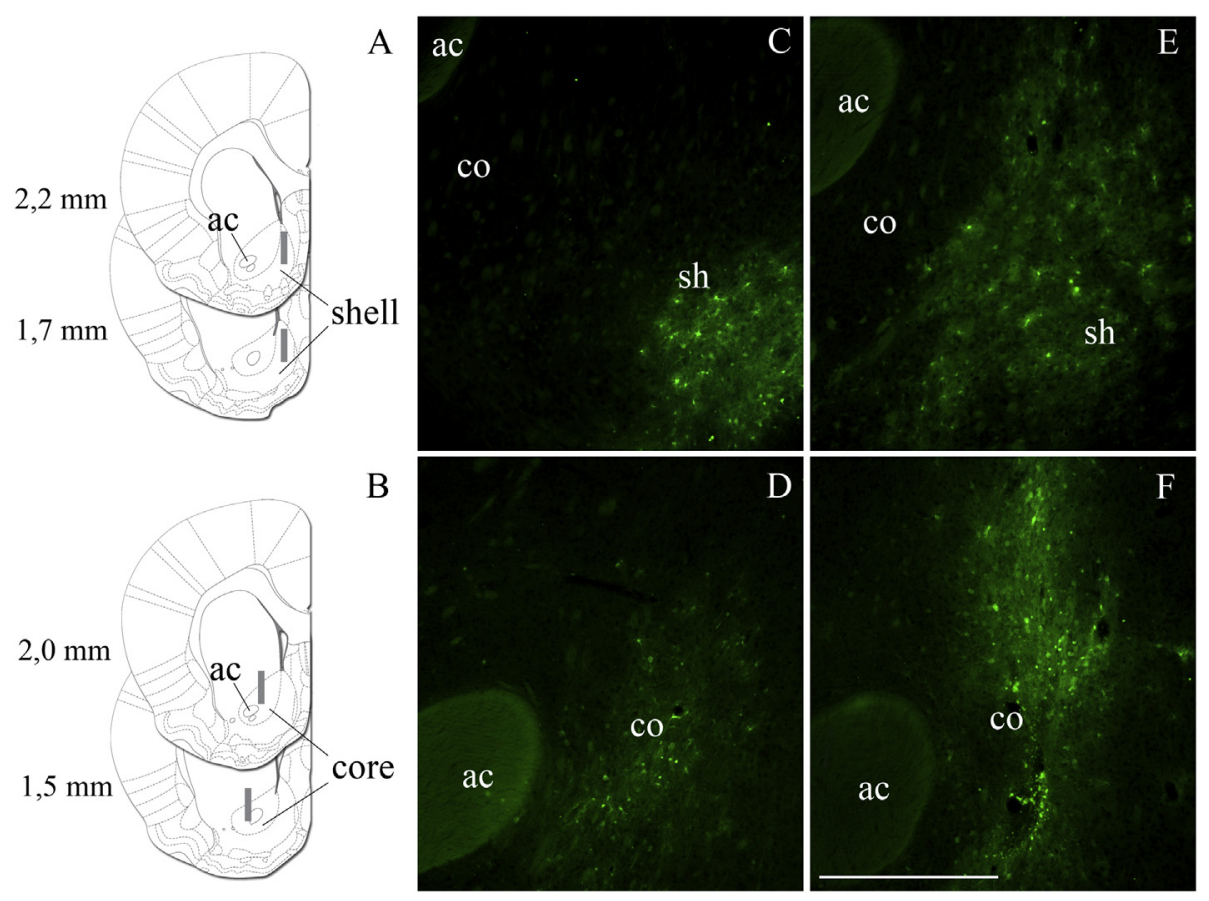

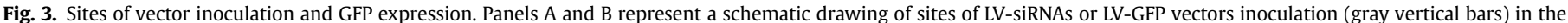

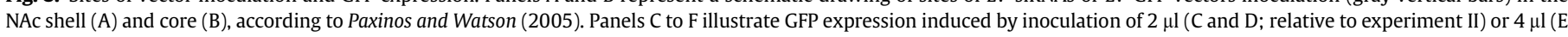

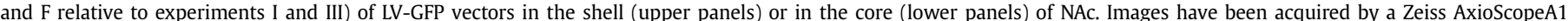
epifluorescent microscope using a $10 \times$ magnification objective, Abbreviations: ac, anterior commissure; co, NAc core; sh, NAc shell. Scale bar is $500 \mu$ m.

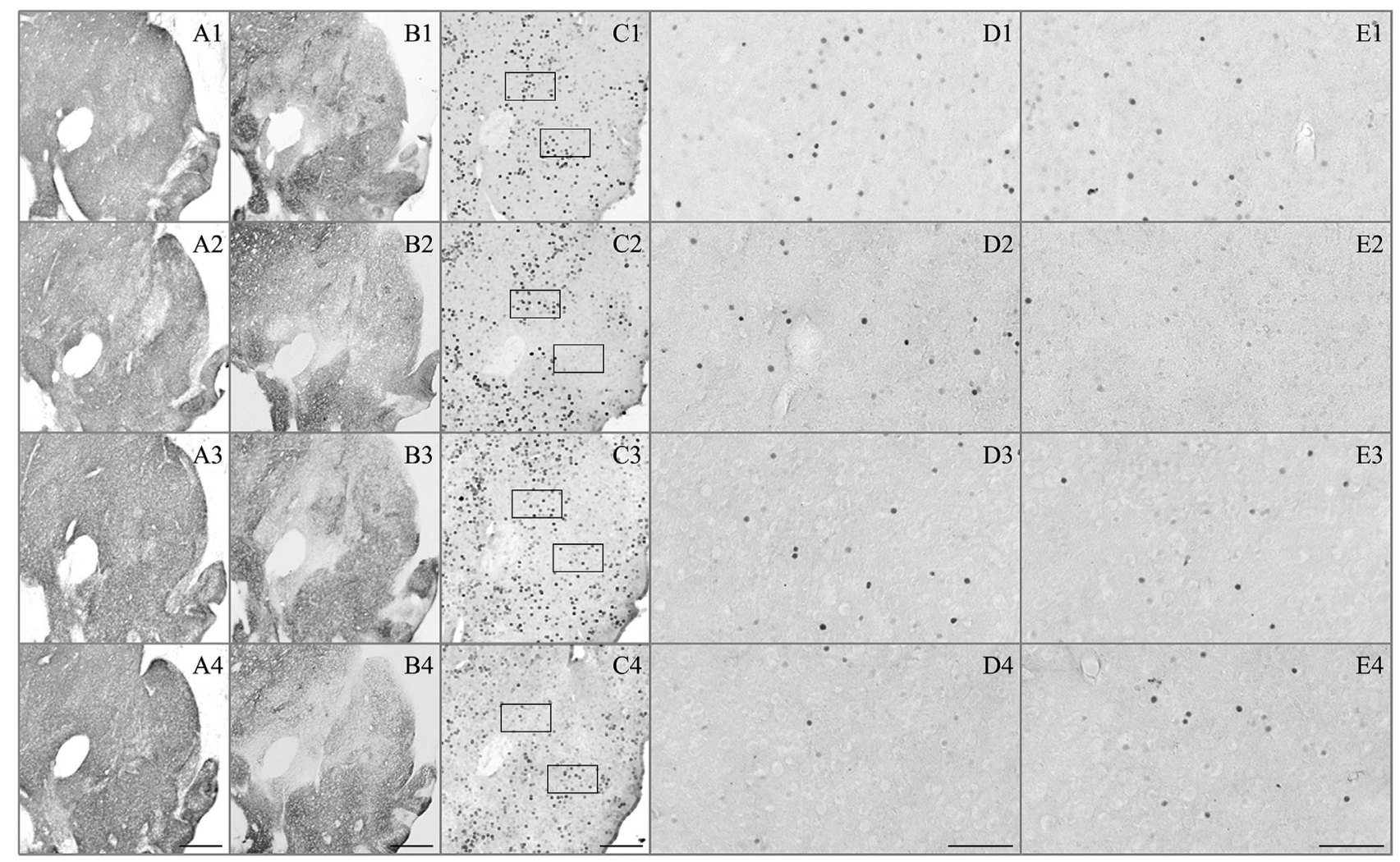

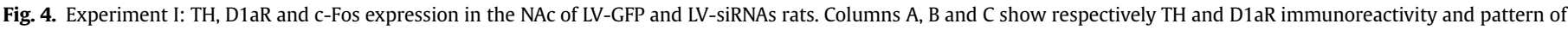

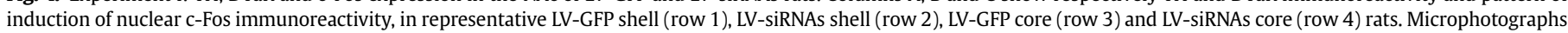

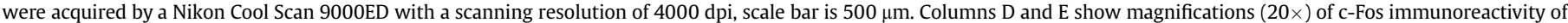

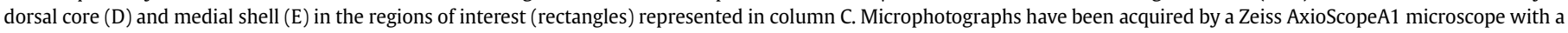
$20 \times$ magnification objective, scale bar is $100 \mu \mathrm{m}$ 
of the LV-GFP shell rats, whereas the LV-siRNAs shell rats showed a reduction of c-Fos positive nuclei (column E) in the NAc shell, particularly in the medial portion compared to the LV-GFP shell control group. Moreover, as illustrated in column B, the same medial portion of the NAc shell of the LV-siRNAs rats showed a decreased D1-IR. Similarly, as shown in lines 3 and 4, sites of inoculation in the NAc core showed lower density of c-Fos positive nuclei and lower D1-IR in the LV-siRNAs core compared to the LVGFP control rats. In a few animals this reduction in D1-IR was also detectable in regions adjacent to the shell or core boundaries, suggesting that excessive spreading of LV-siRNAs vectors may occur with $4 \mu$ l of solution.

Fig. 5 shows percentage changes of D1-IR in the inoculated areas. Three-way ANOVA among group, area and ROI factors showed main effect of group $\left(F_{1,62}=44.99, P=0.000001\right)$ factor. Furthermore, there were significant area $\times$ ROI $\left(F_{1,62}=34.00\right.$, $P=0.000001)$ and group $\times$ area $\times R O I\left(F_{1,62}=55.30, P=0.000001\right)$ interactions, in accordance with a reduction of D1-IR in the inoculated area detectable in both the LV-siRNAs groups compared to their respective LV-GFP controls. As shown in panel 5A, D1-IR was significantly reduced in the NAc shell of the LV-siRNAs shell rats $(79.7 \% \pm 0.9)$ compared to the respective LV-GFP shell control group $(105.1 \% \pm 4.3 ; P<0.05$, Tukey's HSD post-hoc test), while no changes were found in the NAc core in both the LV-siRNAs and LVGFP shell rats. On the other hand, as shown in Fig. 5B, D1-IR was significantly reduced in the NAc core of the LV-siRNAs core rats $(76.7 \% \pm 2.8)$ compared to the respective LV-GFP core control group (108.9\% $\pm 3.4 ; P<0.05$, Tukey's HSD post-hoc test), while no changes were found in the NAc shell in both the LV-siRNAs and LVGFP core rats.

Fig. 5 also shows changes in expression of c-Fos positive nuclei, induced by amphetamine challenge $(5 \mathrm{mg} / \mathrm{kg}$, i.p.) and measured in the NAc shell and core of each experimental group. Four-way ANOVA among challenge, group, area and ROI factors showed main effects of challenge $\left(F_{1,54}=181.02, P=0.000001\right)$ and group $\left(F_{1,54}=6.30, P=0.01\right)$ factors on c-Fos expression. Furthermore, there were significant group $\times$ area $\times$ ROI $\left(F_{1,54}=7.67, P=0.008\right)$ and challenge $\times$ group $\times$ area $\times \operatorname{ROI}\left(F_{1,54}=8.71, P=0.005\right)$ interactions, in accordance with a greater expression of c-Fos positive nuclei elicited by amphetamine challenge in all groups and ROIs,
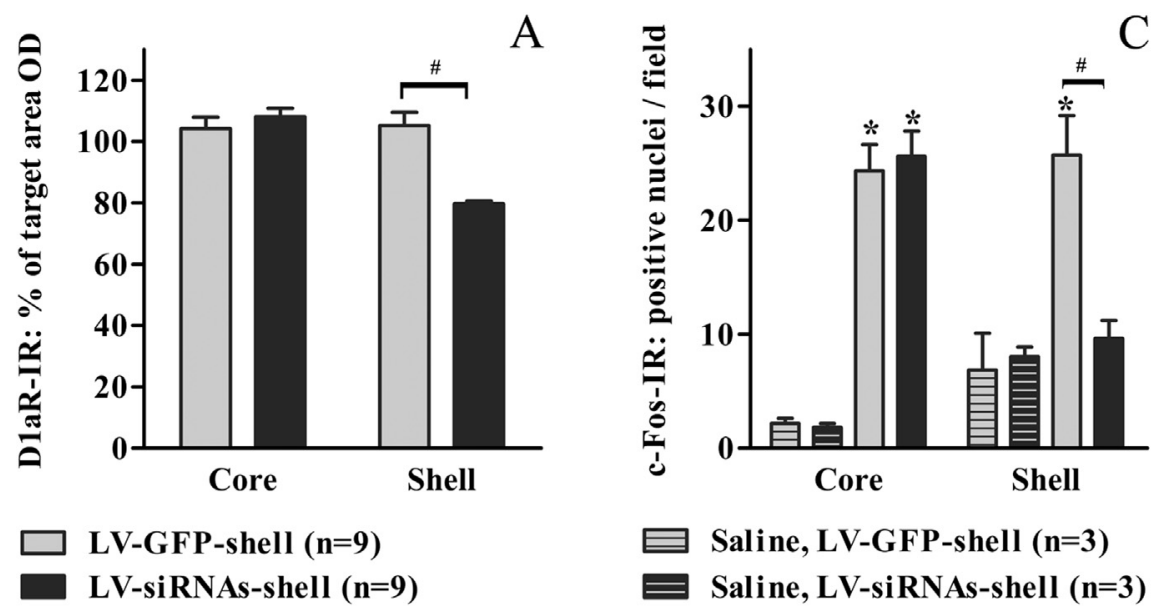

Saline, LV-GFP-shell $(n=3)$

Saline, LV-si RNAs-shell $(n=3)$

Amph, LV-GFP-shell (n=6)

Amph, LV-siRNAs-shell $(n=6)$
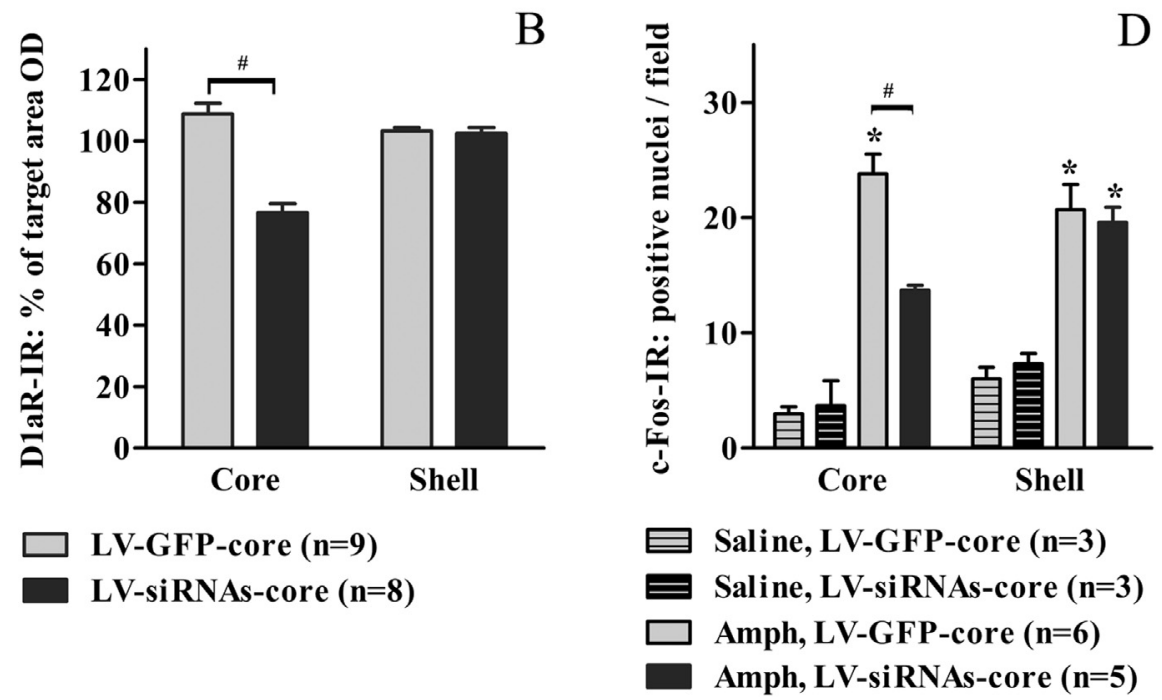

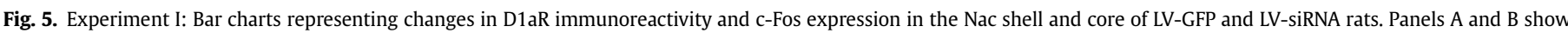

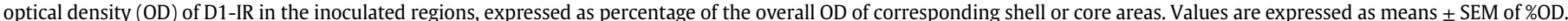

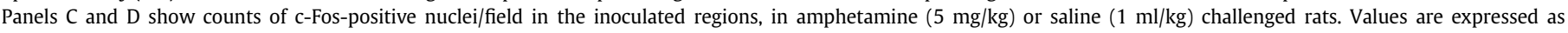
means \pm SEM of positive nuclei/field. *: $P<0.05$ vs respective saline treated group. \#: $P<0.05$ LV-siRNAs $v s$ LV-GFP groups. ANOVA followed by Tukey's HSD post-hoc test. 
compared to the saline treated rats. As shown in panel $5 \mathrm{C}$, expression of c-Fos positive nuclei induced by amphetamine challenge was significantly lower in the NAc shell of the LV-siRNAs shell rats $(9.6 \pm 1.6)$ compared to the respective LV-GFP shell control group (25.7 $\pm 3.5 ; P<0.05$, Tukey's HSD post-hoc test), while such increase was similar in the NAc core for both the LV-siRNAs and LVGFP shell rats. Accordingly, as shown in Fig. 5D, lower levels of c-Fos activation were seen in the NAc core of the LV-siRNAs core rats $(13.7 \pm 0.4)$ compared to the LV-GFP core controls $(23.8 \pm 1.7$; $P<0.05$, Tukey's HSD post-hoc test).

\subsection{Experiment II}

Since in some subjects of Experiment I a reduction of D1-IR was observed in regions outside shell or core boundaries, in order to reduce spreading of LV particles outside the NAc shell and core target regions, a second experiment was performed with two bilateral infusions of $2 \mu \mathrm{l}$ each at a rostrocaudal distance of $500 \mu \mathrm{m}$.

\subsubsection{Cocaine self-administration}

A total of 28 rats were bilaterally inoculated with a double injection of lentiviral vectors in the NAc shell or core compartments and trained to acquire 1-h daily i.v. cocaine SA (unit dose $0.25 \mathrm{mg}$ / $\mathrm{kg}, 5$ day/week), for 15 sessions under FR-1 and 18 sessions under FR-5 schedules of responding, followed by 7 sessions of extinction (saline, $24 \mu \mathrm{l} /$ infusion).

3.2.1.1. Responding. Fig. 6 shows cumulative active and inactive nose-pokes during each i.v. cocaine SA session (unit dose $0.25 \mathrm{mg} /$ kg 1-h daily, 5 days/week) by the LV-siRNAs shell, LV-GFP shell, LVsiRNAs core and LV-GFP core groups, recorded during the FR-1 (1st-15th sessions) and FR-5 (16th-33rd sessions) cocaine SA and extinction (under FR-5, 34th-40th sessions).

The FR- 5 schedule of responding was extended ( 18 sessions), in order to establish if longer cocaine SA exposure could reduce the behavioral differences observed in Experiment I between the LVsiRNAs shell and LV-GFP shell rats; however, even with a longer maintenance period, the NAc shell Drd1 a silenced group (LV-siRNAs shell) showed reduced responding compared to the LV-GFP shell control group, while D1aR knock-down in the NAc core (LV-siRNAs core) once again did not affect behavior compared to the control rats (LV-GFP core).

Four-way ANOVA applied to nose-poke, group, area and session factors showed a main effect of nose-pokes (active vs inactive; $\left.F_{1,32}=129.23, P=0.000001\right)$ and session $\left(F_{39,1248}=31.61\right.$, $P=0.000001)$, and significant group $\times$ area $\left(F_{1,32}=5.55, P=0.02\right)$, group $\times$ nose pokes $\left(F_{1,32}=5.03, P=0.03\right)$, group $\times$ session $\left(F_{39,1248}=2.80, P=0.000001\right)$, nose pokes $\times \operatorname{session}\left(F_{39,1248}=29.30\right.$, $P=0.000001)$, group $\times$ area $\times$ nose pokes $\left(F_{1,32}=6.75, P=0.01\right)$, group $\times$ area $\times$ session $\left(F_{39,1248}=4.44, P=0.000001\right)$, group $\times$ nose pokes $\times$ session $\left(F_{39,1248}=2.37, P=0.00006\right)$, area $\times$ nose pokes $\times \operatorname{session}\left(F_{39,1248}=1.49, P=0.03\right)$ and group $\times$ area $\times$ nosepokes $\times$ session $\left(F_{39,1248}=4.90, P=0.000001\right)$ interactions.

As shown in Fig. 6, the LV-GFP shell (panel A) and all the core groups (panel B) acquired cocaine SA under FR-1 schedule during the second week and increased active nose-poking behavior starting from the 16th session under FR- 5 schedule of responding $(P<0.05$ for active nose pokes compared to inactive ones, Tukey's HSD post-hoc test). On the other hand, active nose-pokes emitted by LV-siRNAs shell rats were not significantly higher compared to inactive ones, even during the extinction phase, in which there is usually a tendency to observe a further increase in nose poking
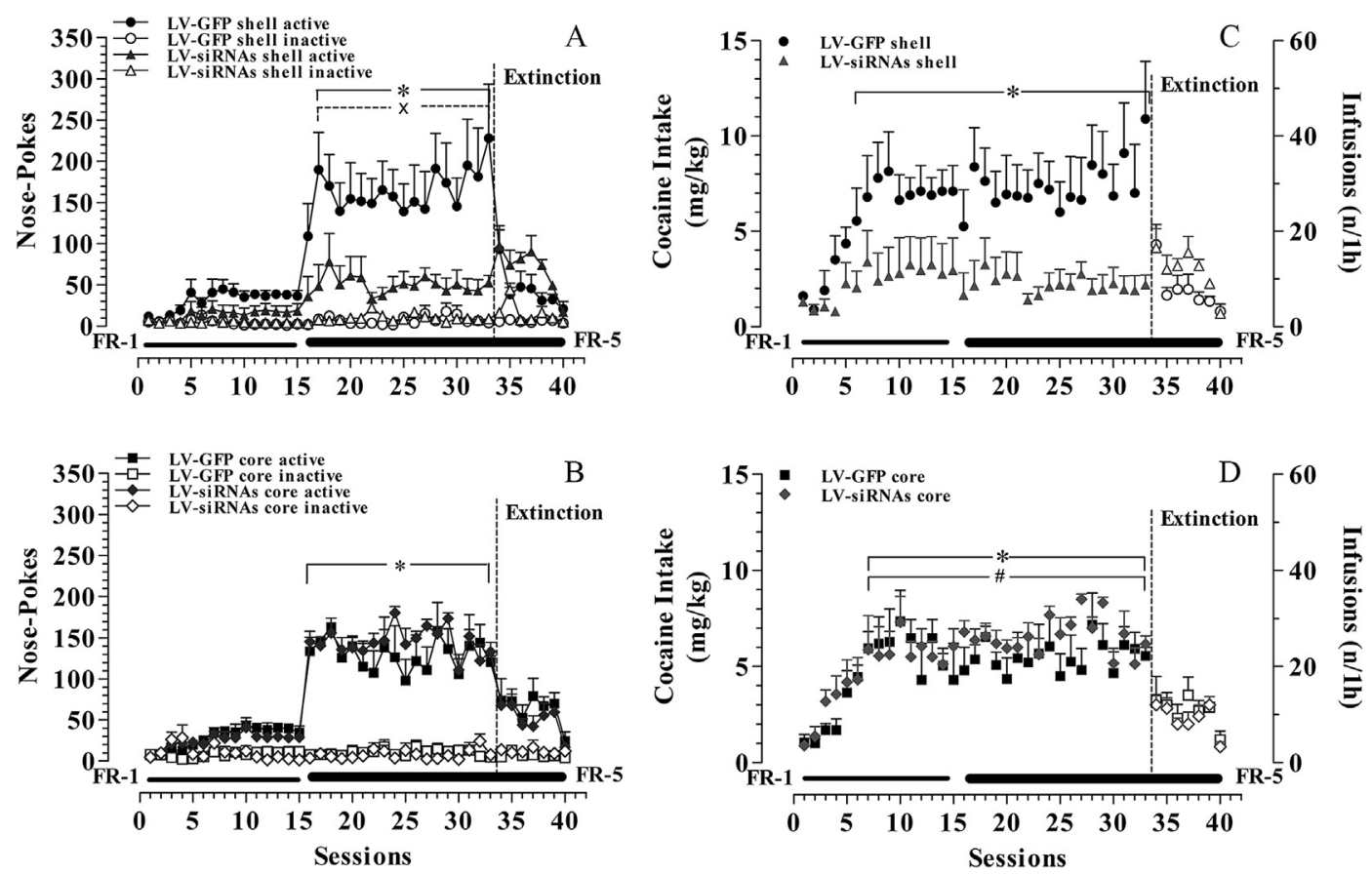

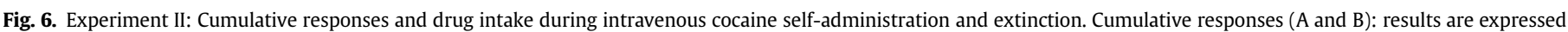

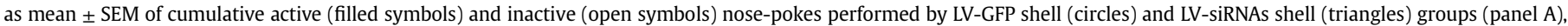

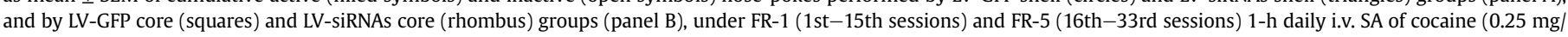

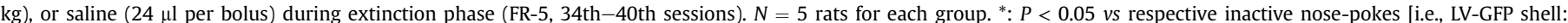

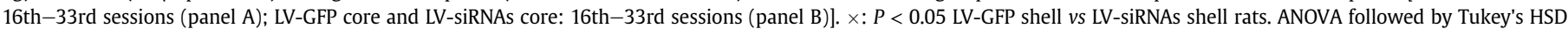

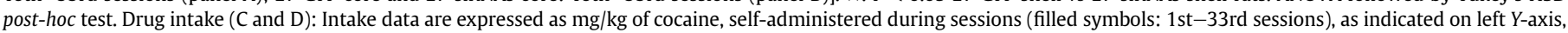

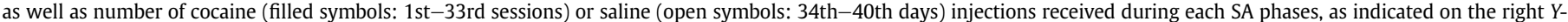

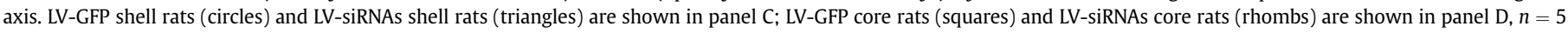
rats for each group. ${ }^{*}: P<0.05$ vs session 1 within group; ANOVA followed by Fisher's LSD post-hoc test. 
activity. Moreover, Tukey's HSD post-hoc test showed higher active nose poking activity $(P<0.05)$ of the LV-GFP shell group under FR-5 schedule (16th-33rd sessions) compared to the LV-siRNAs shell rats (Fig. 6A). Conversely, the LV-siRNAs core rats showed behavioral responses similar to those performed by the LV-GFP core group, in all phases of i.v. cocaine SA (Fig. 6B).

3.2.1.2. Cocaine intake. Three-way ANOVA applied to daily infusions (cocaine or saline) showed a main effect of session $\left(F_{39,624}=10.92, P=0.000001\right)$ and group $\times$ area $\left(F_{1,16}=7.14\right.$, $P=0.02)$, group $\times$ session $\left(F_{39,624}=1.79, P=0.003\right)$ and group $\times$ area $\times$ session $\left(F_{39,624}=2.73, P=0.000001\right)$ interactions.

As shown in Fig. 6, daily infusions and cocaine intake increased in the LV-GFP shell group (Fig. 6C, $P<0.05$ on 6th-33rd sessions compared to 1st week, Fisher's LSD post-hoc test) but not in the LVsiRNAs shell rats. As in Experiment I, the LV-siRNAs core rats showed a pattern of daily infusions not different from the LV-GFP core rats (Fig. 6D). Three-way ANOVA applied to cocaine intake, calculated as weekly means, showed a main effect for group $\left(F_{1,16}=5.43, P=0.03\right)$, for week $\left(F_{6,96}=10.60, P=0.000001\right)$ and group $\times$ area interactions $\left(F_{1,16}=8.17 P=0.01\right)$. Post-hoc analysis indicated an increase in cocaine intake over the weeks in all experimental groups, except for LV-siRNAs shell rats (Table 2).

\subsubsection{Immunohistochemistry}

3.2.2.1. GFP expression. In vivo transfection, as indicated by GFP expression, affected an area of about $150-200 \mu \mathrm{m}$ of radius for each $2-\mu$ inoculation (Fig. 3, panels C and D).

3.2.2.2. TH expression. As shown in Fig. 7 (panels A1-A4) the integrity of the presynaptic dopaminergic fibers in the NAc core and shell was preserved, except for a slight scar along the track of the injection cannula.

3.2.2.3. D1aR and c-Fos expression. D1-IR and c-Fos-IR in the NAc shell and core of representative rats from the Experiment II are shown in Fig. 7. Column B shows D1-IR, column C shows the pattern of distribution of nuclear c-Fos-IR, while columns D and E are $20 \times$ magnifications of c-Fos-IR of the selected rectangular regions indicated in column $\mathrm{C}$, relative to dorsal core and medial shell respectively. Similar to Experiment I, c-Fos expression was reduced in the inoculated area for both the LV-siRNAs groups compared to the respective LV-GFP controls. Moreover, the same inoculated areas of LV-siRNAs rats were characterized by a decreased D1-IR, as illustrated in column B.

\section{Table 2}

Experiment II. Mean \pm SEM of weekly cocaine intake per session $(\mathrm{mg} / \mathrm{kg})$ in LV-GFP and LV-siRNAs shell and core groups under FR-1 (1st-3rd week) and FR-5 (4th-7th week) i.v. cocaine SA. $N=5$ rats per group.*: $P<0.05$ vs 1 st week; $\times: P<0.05$ vs respective GFP control group. ANOVA followed by LSD's post-hoc test.

\begin{tabular}{cllll}
\hline SA phases: & Groups: & & \\
\cline { 2 - 5 } & $\begin{array}{l}\text { LV-GFP } \\
\text { shell }\end{array}$ & $\begin{array}{l}\text { LV-siRNAs } \\
\text { shell }\end{array}$ & $\begin{array}{l}\text { LV-GFP } \\
\text { core }\end{array}$ & $\begin{array}{l}\text { LV-siRNAs } \\
\text { core }\end{array}$ \\
\hline FR-1 & & & & \\
1st week & $2.5 \pm 0.3$ & $1.3 \pm 0.3$ & $1.8 \pm 0.4$ & $2.6 \pm 0.7$ \\
2nd week & $7.0 \pm 1.6^{*}$ & $2.7 \pm 1.4^{\times}$ & $6.1 \pm 1.2^{*}$ & $5.7 \pm 1.8^{*}$ \\
3rd week & $7.0 \pm 1.1^{*}$ & $3.0 \pm 1.6^{\times}$ & $5.3 \pm 0.9^{*}$ & $5.7 \pm 0.9^{*}$ \\
FR-5 & & & & \\
4th week & $6.9 \pm 1.6^{*}$ & $2.4 \pm 1.2^{\times}$ & $5.3 \pm 0.7^{*}$ & $6.4 \pm 0.7^{*}$ \\
5th week & $6.9 \pm 1.5^{*}$ & $2.0 \pm 0.6^{\times}$ & $6.1 \pm 0.7^{*}$ & $6.5 \pm 0.7^{*}$ \\
6th week & $7.4 \pm 2.0^{*}$ & $2.2 \pm 0.6^{\times}$ & $5.8 \pm 1.0^{*}$ & $7.2 \pm 0.4^{*}$ \\
7th week & $9.0 \pm 2.5^{*}$ & $2.0 \pm 0.6^{\times}$ & $5.9 \pm 0.6^{*}$ & $6.0 \pm 1.0^{*}$ \\
\hline
\end{tabular}

*: $P<0.05$ vs 1 st week.

${ }^{\times}:<0.05$ vs respective GFP control group.
Fig. 8 shows percentage changes of D1-IR in inoculated areas. Values from the two bilateral sites of vector inoculation were averaged for each subject. Three-way ANOVA with group, area and $R O I$ as factors showed a main effect of group $\left(F_{1,32}=44.05\right.$, $P=0.000001)$ and an interaction of area $\times R O I\left(F_{1,32}=42.98\right.$, $P=0.000001)$ and group $\times$ area $\times R O I\left(F_{1,32}=39.75, P=0.000001\right)$. As shown in panel 8A, D1-IR was significantly reduced in the NAC shell of LV-siRNAs shell rats $(78.4 \% \pm 3.6)$ compared to the respective LV-GFP shell control group $(105.6 \% \pm 3.4 ; P<0.05$, Tukey's HSD post-hoc test), while no changes were found in the NAc core in both the LV-siRNAs and LV-GFP shell rats. Accordingly, as shown in Fig. 8B, D1-IR was significantly reduced in the NAc core of the $\mathrm{LV}$-siRNAs core rats $(79.4 \% \pm 3.5)$ compared to the respective LV-GFP core control group $(109.5 \% \pm 2.4 ; P<0.05$, Tukey's HSD post-hoc test), while no changes were found in the NAc shell in both the LV-siRNAs and LV-GFP core rats.

Fig. 8 also shows changes in expression of c-Fos positive nuclei, induced by amphetamine challenge ( $5 \mathrm{mg} / \mathrm{kg}$, s.c.), measured in the inoculated areas of NAc shell and core of each experimental group. Three-way ANOVA among group, area and ROI factors showed main effect of group $\left(F_{1,32}=36.06, P=0.000001\right)$ factor on $c$-Fos expression. Furthermore there were significant area $\times$ ROI $\left(F_{1,32}=42.88, P=0.000001\right)$ and group $\times$ area $\times R O I\left(F_{1,32}=21.52\right.$, $P=0.00004)$ interactions. Fig. $8 C$ shows how levels of expression of c-Fos positive nuclei induced by amphetamine challenge were significantly lower in the NAc shell of LV-siRNAs shell rats $(9.5 \pm 1.1)$ compared to the respective LV-GFP shell control group $(23.2 \pm 1.5$; $P<0.05$, Tukey's HSD post-hoc test), while such increase was similar in the NAc core for both the LV-siRNAs and LV-GFP shell rats. Accordingly, as shown in Fig. 8D, lower levels of c-Fos activation were seen in the NAc core of the LV-siRNAs core rats $(10.9 \pm 1.4)$ as compared to the LV-GFP core controls $(21.9 \pm 1.1 ; P<0.05$, Tukey's HSD post-hoc test).

\subsection{Experiment III}

\subsubsection{Heroin self-administration}

In Experiment III, the effect of bilateral infusion of $4 \mu \mathrm{l}$ of lentiviral vectors in the NAc shell (LV-siRNAs shell and LV-GFP shell groups) on i.v. heroin SA was evaluated. Rats were trained to acquire 1-h daily i.v. heroin SA (unit dose $0.05 \mathrm{mg} / \mathrm{kg}, 5$ day/week) under FR-1 then switched to FR-5 schedules of responding, followed by extinction (saline, $0.48 \mu \mathrm{l} /$ infusion; FR-5).

3.3.1.1. Responding. Fig. 9A shows cumulative active and inactive nose-pokes performed by the LV-GFP and LV-siRNAs groups during FR-1 (1st-10th sessions) and FR-5 (11st-25th sessions) heroin SA as well as the extinction (26th-35th sessions, FR-5) phase.

Three-way ANOVA with nose-pokes (active and inactive), group (LV-GFP and LV-siRNAs) and session (days) as factors, showed a main effect of nose-pokes (active vs inactive; $F_{1,34}=148.89$, $P=0.000001)$ and session $\left(F_{34,1156}=32.14, P=0.000001\right)$ and interaction of nose-poke $\times$ session $\left(F_{34,1156}=14.92, P=0.000001\right)$, but not of group $\left(F_{1,34}=0.02, P=0.88, N\right.$.S. $)$, group $\times$ session $\left(F_{34,1156}=1.26, P=0.14\right.$, N.S. $)$ and group $\times$ nose-poking $\times$ session $\left(F_{34,1156}=0.76, P=0.84\right.$, N.S. $)$ interactions. Both the LV-GFP and LVsiRNAs groups acquired heroin SA behavior under FR-1 schedule during the second week and further increased active nose poking under FR-5 $(P<0.05$ active $v s$ inactive nose-pokes, Tukey's HSD post-hoc test). Under extinction, substitution of heroin with saline induced an increase of responding during the 1 st day followed by a progressive decrease in both experimental groups (Fig. 9A).

Thus, infusion of LV-siRNAs in the NAc shell did not affect heroin SA behavior compared to the LV-GFP shell rats. 


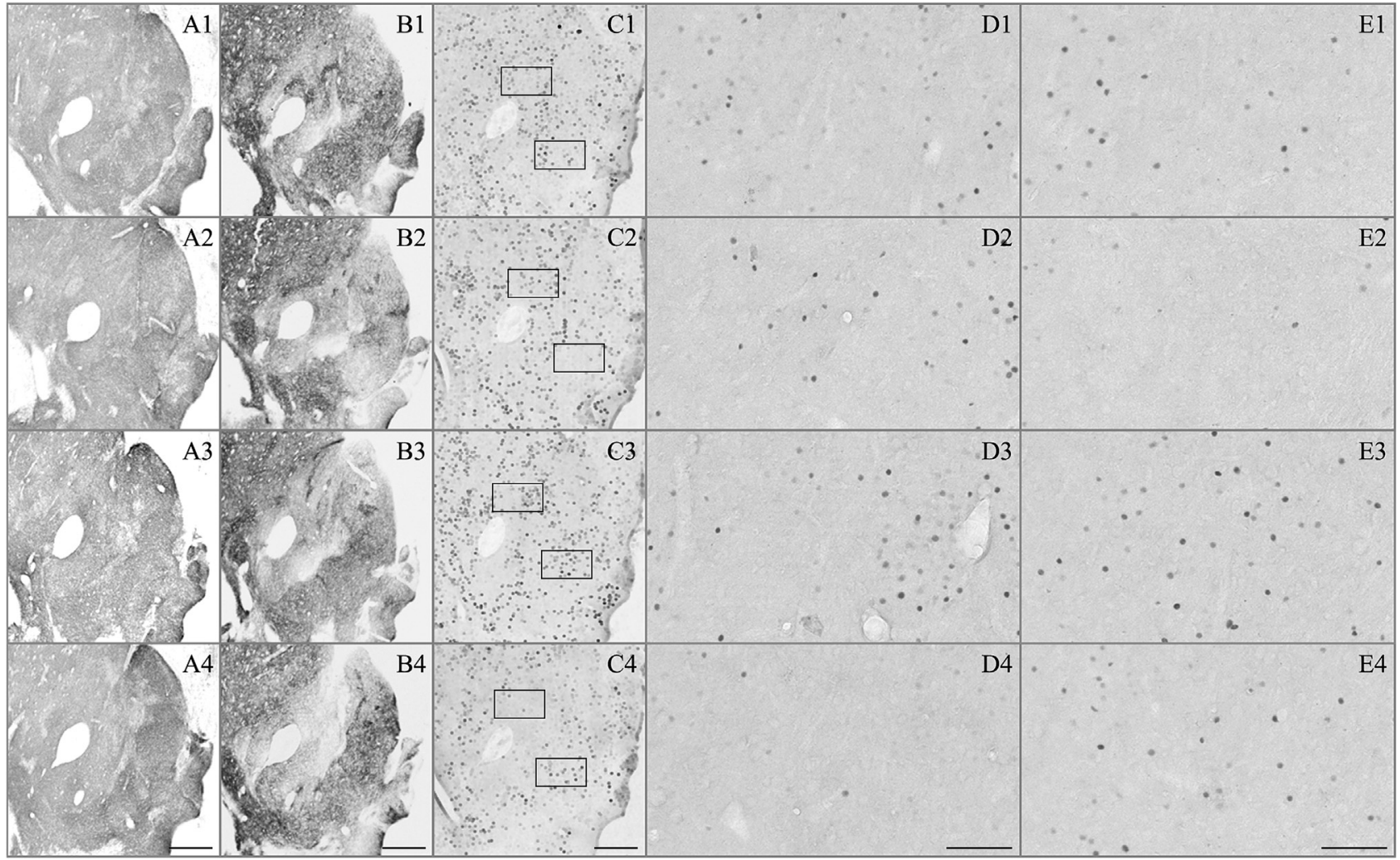

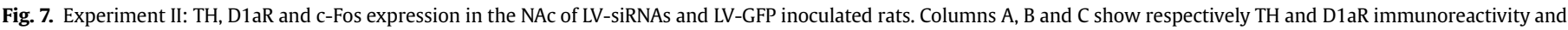

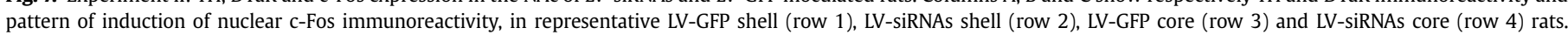

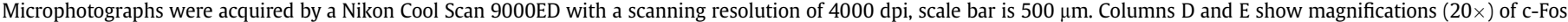

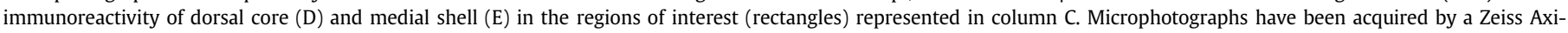
oScopeA1 microscope with a $20 \times$ magnification objective, scale bar is $100 \mu \mathrm{m}$.

3.3.1.2. Heroin intake. Two-way ANOVA, among group and session factors, applied to the daily infusions of heroin (acquisition and maintenance) or saline (extinction) showed main effect of session $\left(F_{34,578}=9.85, P=0.000001\right)$ but not group $\times \operatorname{session}\left(F_{34,578}=1.18\right.$, $P=0.23$, N.S.) interaction.

Fig. 9B shows the number of heroin infusions in the LV-GFP and LV-siRNAs rats; during the 1 st day of extinction saline injections significantly increased in both groups.

On the other hand, two-way ANOVA applied to heroin intake expressed as weekly means, showed a main effect of week $\left(F_{4,68}=3.93, P=0.01\right)$, and an increase of heroin intake during the 2nd and the 4th weeks in the LV-siRNAs but not in the LV-GFP rats, and a slight but non-significant reduction during the 3rd week in both experimental groups (Table 3 ).

\subsubsection{Immunohistochemistry}

3.3.2.1. GFP expression. As observed in Experiment I, in vivo transfection, indicated by GFP expression, affected an area of about $350 \mu \mathrm{m}$ of radius around the site of inoculation of $4 \mu \mathrm{l}$ of LV-GFP (Fig. 3, panels E and F).

3.3.2.2. D1aR and c-Fos expression. D1aR and c-Fos immunoreactivity in the NAc shell of representative rats from the Experiment III are shown in Fig. 10. Column A shows D1-IR, column B represents the pattern of nuclear c-Fos-IR distribution, while column $C$ shows $20 \times$ magnifications of c-Fos-IR of the selected rectangular regions indicated in column $\mathrm{B}$, relative to the medial shell. c-Fos expression was reduced in the inoculated area of the LV-siRNAs treated animals compared to the respective LV-GFP controls. Moreover, the same inoculated area of LV-siRNAs rats was characterized by a decreased D1-IR, as illustrated in column A.

Fig. 11 shows percentage changes of D1-IR in inoculated areas. D1-IR was reduced in the NAc shell of the LV-siRNAs shell rats $(73.5 \% \pm 6.9)$ compared to the respective LV-GFP shell control group $\left(102.3 \% \pm 2.0\right.$, One-way ANOVA: $F_{1,17}=17.65, P=0.000001$; Fig. 11A). Accordingly, as shown in Fig. 11B, expression of c-Fos positive nuclei induced by amphetamine in the inoculated area was lower in the NAc shell of the LV-siRNAs rats $(9.6 \pm 1.5)$ compared to the LV-GFP control group ( $18.6 \pm 1.7$; one-way ANOVA: $F_{1,17}=15.01$, $P=0.000001)$.

\section{Discussion}

The aim of the present study was to investigate the role of D1aR in cocaine reinforcement as estimated by acquisition of i.v. cocaine SA in rats. In order to selectively silence D1aR expression in the two main subdivisions of the NAc, the shell and the core, lentiviral vectors expressing Drd1a-siRNA were stereotaxically infused in the medial shell and in the dorsal core of the NAc of different groups of rats.

Two different procedures were used. In Experiment I, $4 \mu \mathrm{l}$ of viral stock solution were infused bilaterally into a single site in the NAc shell or core, while in Experiment II, the $4-\mu$ l stock solution was split in two $2-\mu$ l aliquots that were infused bilaterally into two different sites $\approx 500 \mu \mathrm{m}$ apart in the NAc shell or core. This second procedure was chosen in an attempt to improve the site specificity of silencing by restricting it to each NAc subdivision. In fact, histological analysis of Experiment I had shown that infusion of $4 \mu \mathrm{l}$ of 

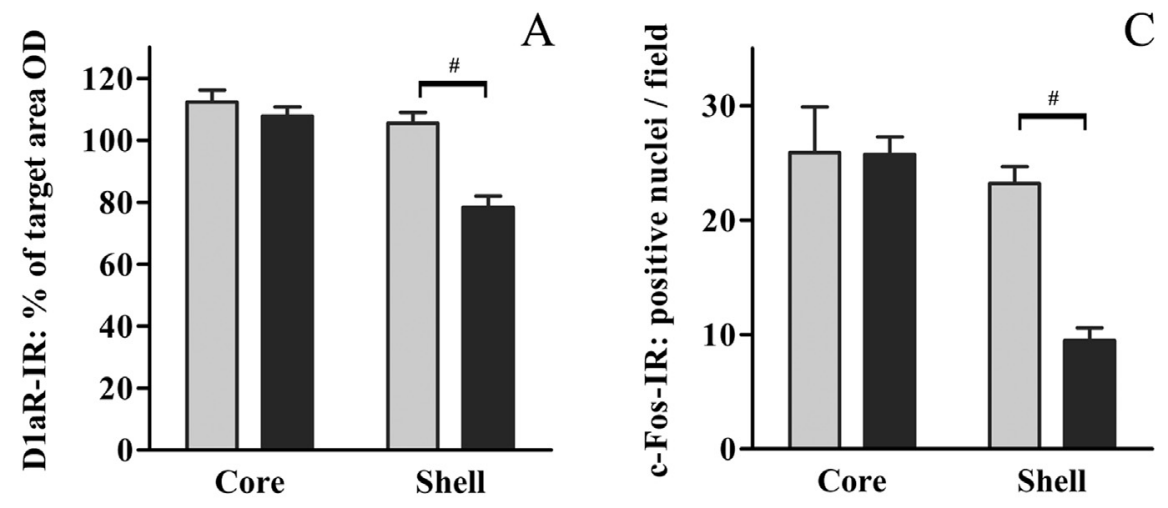

\section{LV-GFP-shell $(\mathrm{n}=5)$ \\ LV-siRNAs-shell $(n=5)$}

\section{LV-GFP-shell ( $\mathrm{n}=5)$}

LV-siRNAs-shell ( $n=5)$
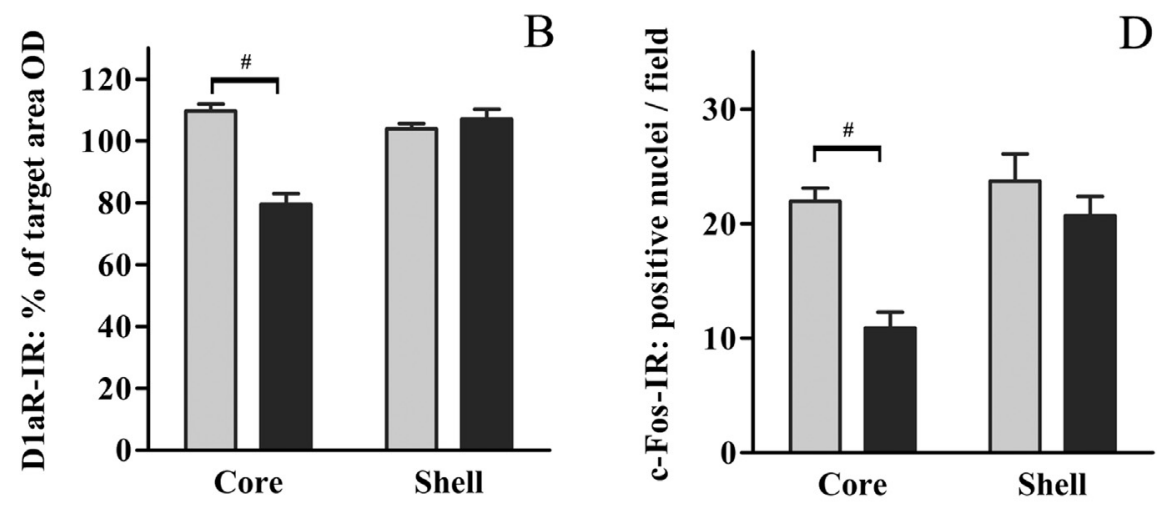

\section{LV-GFP-core $(\mathrm{n}=5)$ \\ LV-siRNAs-core $(n=5)$}

\section{LV-GFP-core $(\mathrm{n}=5)$ \\ LV-siRNAs-core $(n=5)$}

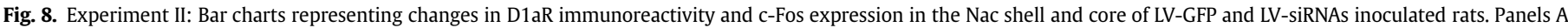

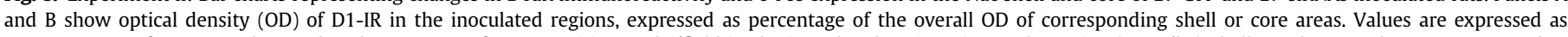

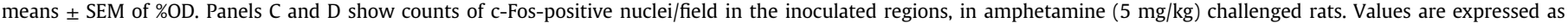
means \pm SEM of positive nuclei/field. \#: $P<0.05$ LV-siRNAs $v s$ LV-GFP groups. ANOVA followed by Tukey's HSD post-hoc test.

viral solution into the medial shell resulted in transfection to the adjacent aspects of the core and olfactory tubercle, instead of just being confined to the medial shell. Histological analysis of Experiment II showed that, indeed, the area transfected ( $\approx 150 \mu \mathrm{m}$ radius) was well within the NAc shell boundaries.

Stimulation of dopamine transmission by psychostimulants induces dose-dependent expression of c-Fos in rat striatum (Brenhouse and Stellar, 2006; Graybiel et al., 1990). This effect is abolished by pretreatment with the D1R antagonist SHC 23390, (Graybiel et al., 1990) and is lost in D1 KO mice (Moratalla et al., 1996). Therefore, stimulation of c-Fos expression by psychostimulants is mediated by stimulation of D1aR. In view of this, c-Fos expression in the NAc shell and core regions induced by amphetamine challenge, was utilized as an estimate of D1aR function. The density of c-Fos positive nuclei was reduced in the shell compartment in LV-siRNAs shell groups in Experiments I, II and III, and in the core compartment of LV-siRNAs core groups in Experiment I and II.

The persistence of a residual D1aR responsiveness might explain the SA behavior recorded in LV-siRNAs shell group in Experiment I. However, in this experiment rats showed a delayed acquisition, a lower active nose poking activity, and a reduced cocaine intake, compared to LV-GFP shell control rats. On the other hand, in Experiment II, in which the total volume of $4 \mu \mathrm{l}$ was infused into two sites along the rostro-caudal axis, rats did not acquire cocaine $\mathrm{SA}$, as significant differences between active and inactive nosepokes were not reached, neither under FR-1 nor under FR-5 schedule of responding. Infusion of control GFP-expressing vectors in the NAc shell did not affect acquisition. On the other hand, silencing of D1aR expression in the NAc core did not affect acquisition of responding for i.v. cocaine. In both experiments, the group infused with LV-siRNAs for D1aR in the shell showed a reduction of responding for cocaine and of cocaine intake compared to controls, while infusion of the same LV-Drd1a-siRNAs in the NAc core did not affect responding for cocaine. Therefore, only D1aR silencing in the NAc shell was able to impair acquisition of responding for i.v. cocaine SA, indicating that DA D1aRs in the NAc shell, but not in the core, are important for acquisition of cocaine reinforcement. Silencing of NAc shell D1aR expression by infusion of LV-siRNA in the NAc shell using a protocol similar to that utilized in Experiment I, failed to affect acquisition of heroin i.v. SA. This observation, consistent with previous studies on the effect of DA receptor antagonists on the acquisition of heroin SA (Ettenberg et al., 1982), excludes the possibility that impairment of cocaine SA induced by 

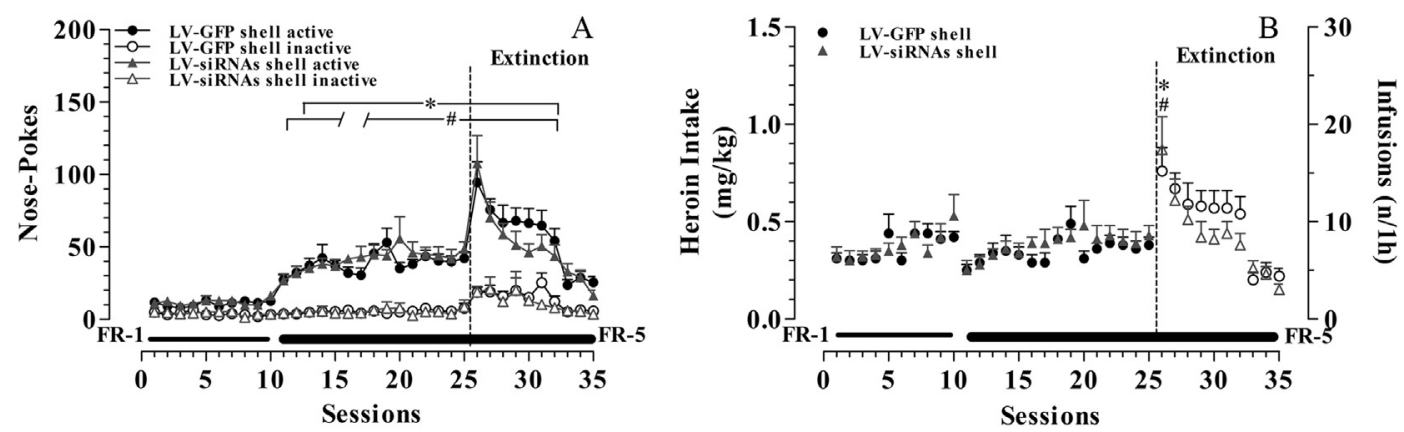

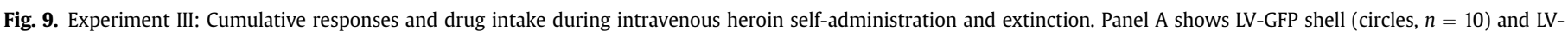

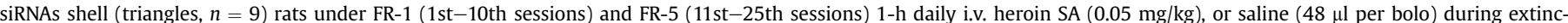

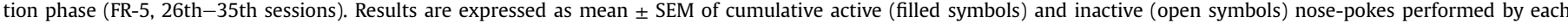

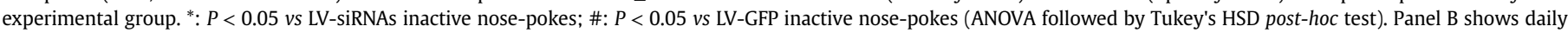

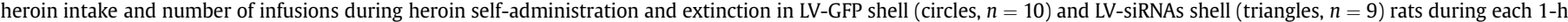

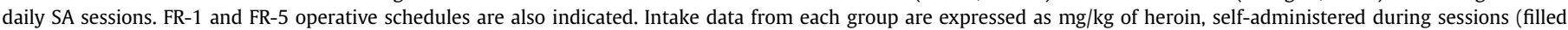

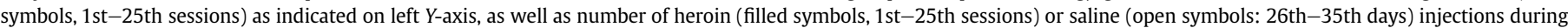

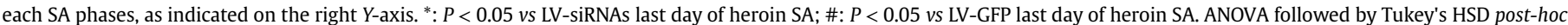
test.

silencing of NAc shell DA D1aR is due to a general impairment of instrumental learning or motivation. Collectively therefore, the impairment of cocaine self-administration, elicited by silencing of $\mathrm{D} 1 \mathrm{aR}$ expression in the NAc shell, can be interpreted as due to a devaluation of the reinforcing properties of cocaine.

Previous studies comparing the role of NAc shell and core DA in cocaine reinforcement have obtained contrasting results. Bari and Pierce (2005) reported that both intra-shell and intra-core infusion of D1 (SCH23390) and D2 (eticlopride) receptor antagonists reduce the breaking point in a progressive ratio schedule of i.v. cocaine self-administration. However, D1 and D2 antagonists also reduced food reinforcement when infused in the NAc core, while they did not affect it after infusion in the shell. Therefore, it was concluded that blockade of DA receptors in the NAc core impaired cocaine reinforcement by a general impairment of motivation.

The possibility that blockade of DA receptors in the NAc core by local DA antagonists impairs cocaine reinforcement, due to an effect on motivation, is also suggested by Di Ciano (2008), who reported that infusion of flupentixol in the core and in the mediodorsal part of the shell reduced responding for cocaine on a second-order schedule of i.v. self-administration. In contrast, on an FR-1 schedule flupentixol increased responding when infused in the shell while decreased it after infusion in the core. These observations were interpreted to indicate that DA in the NAc shell, rather than in the core, mediates cocaine reward (Di Ciano, 2008).

Recently, Veeneman et al. (2012) reported that the infusion of flupentixol in the portion of the shell just ventral to the core, decreases FR-1 responding for i.v. cocaine self-administration, as well

\section{Table 3}

Experiment III. Mean \pm SEM of weekly heroin intake per session $(\mathrm{mg} / \mathrm{kg})$ in LV-GFP $(N=9)$ and LV-siRNAs $(N=10)$ groups under FR-1 (1st-2nd week) and FR-5 (3rd-5th week) i.v. heroin SA.* $P<0.05$ vs 1 st week. ANOVA followed by LSD's post-hoc test.

\begin{tabular}{lll}
\hline SA phases: & \multicolumn{2}{l}{ Groups: } \\
\cline { 2 - 3 } & LV-GFP shell & LV-siRNAs shell \\
\hline FR-1 & $0.33 \pm 0.03$ & $0.32 \pm 0.02$ \\
1st week & $0.36 \pm 0.03$ & $0.48 \pm 0.05^{*}$ \\
2nd week & & \\
FR-5 & $0.31 \pm 0.03$ & $0.31 \pm 0.04$ \\
3rd week & $0.30 \pm 0.04$ & $0.44 \pm 0.07^{*}$ \\
4th week & $0.37 \pm 0.03$ & $0.41 \pm 0.05$ \\
5th week & & \\
\hline
\end{tabular}

*: $P<0.05$ vs 1 st week. as sucrose reinforcement, and attributed these effects to an impairment of motivation. Infusion of flupentixol into the core had no effect. The observations of Veeneman et al. (2012) are at odds with those of Bari and Pierce (2005) (no effect of intra-shell DA antagonists on food reinforcement) and with those of Bachtell et al. (2005) and Di Ciano (2008) (increase in responding for i.v. cocaine after intra-shell DA antagonists). Indeed, Veeneman et al. (2012) attributed to NAc shell DA the motivational role that Bari and Pierce (2005) and Di Ciano (2008) attributed to NAc core DA. It is notable however, that in the study of Veeneman et al. (2012), in order to infuse flupentixol in the ventro-medial shell, cannulas went through the dorsally located core, while in the studies of Bari and Pierce (2005), Bachtell et al. (2005) and Di Ciano (2008), as well as in the present study, the medio-dorsal part of the shell was targeted, thus sparing the core. In order to explain the above discrepancies, one might consider that in the study of Veeneman et al. (2012), flupentixol may have reached the dorsally located core by leakage of the fluid along the track that the cannula left in the core on its way to the shell. In view of this, and of the fact that food (Bari and Pierce, 2005) or heroin reinforcement (Alderson et al., 2001 and present study) were not affected by excitotoxic lesions of the shell (Alderson et al., 2001), as well as more medial intra-shell placements of D1 antagonists (Bari and Pierce, 2005) and of LVDrd1a-siRNAs (present study) that spare the core, we suggest that the effect of the intra-shell flupentixol on cocaine and food reinforcement reported by Veeneman et al. (2012) could be due to blockade of DA receptors of the core. Viewed from this perspective, the results of Veeneman et al. (2012) can be reconciled with those of previous studies and of the present one. Veeneman et al. (2012) also reported that intra-shell infusion of flupentixol at the beginning of each of the 5 trials impairs acquisition of cocaine selfadministration. However, as soon as flupentixol was discontinued responding increased to the levels of controls. On this basis, it was suggested that the drug had not impaired acquisition but responding itself. This effect, however, may have originated from the core rather than the shell (see above).

In relation to the main issue of the present study, i.e. to clarify the relative role of NAc shell and core DA D1aR in cocaine reinforcement, the use of LV-RNAi approach to impair receptor function shows two main advantages over local intracerebral infusion of specific receptor antagonists. The first advantage is that LV-RNAi can be applied to chronic behavioral paradigms, in which the repeated intracerebral infusion of highly lipophilic drugs would result in drug diffusion to large brain areas, if not to the whole 


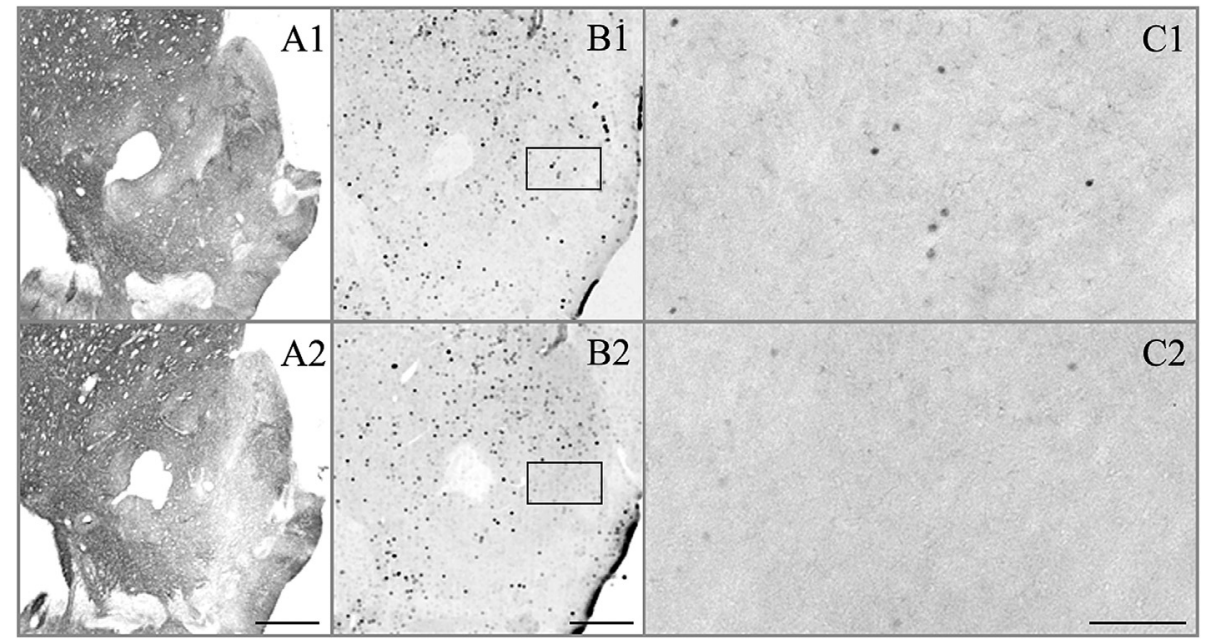

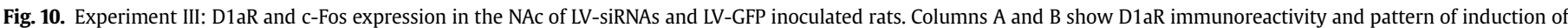

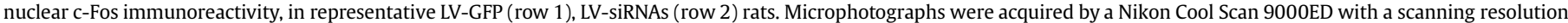

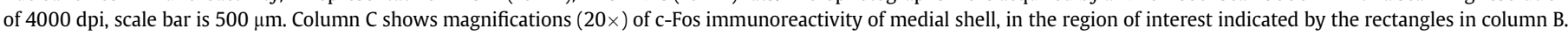
Microphotographs have been acquired by a Zeiss AxioScopeA1 microscope with a $20 \times$ magnification objective, scale bar is $100 \mu \mathrm{m}$.
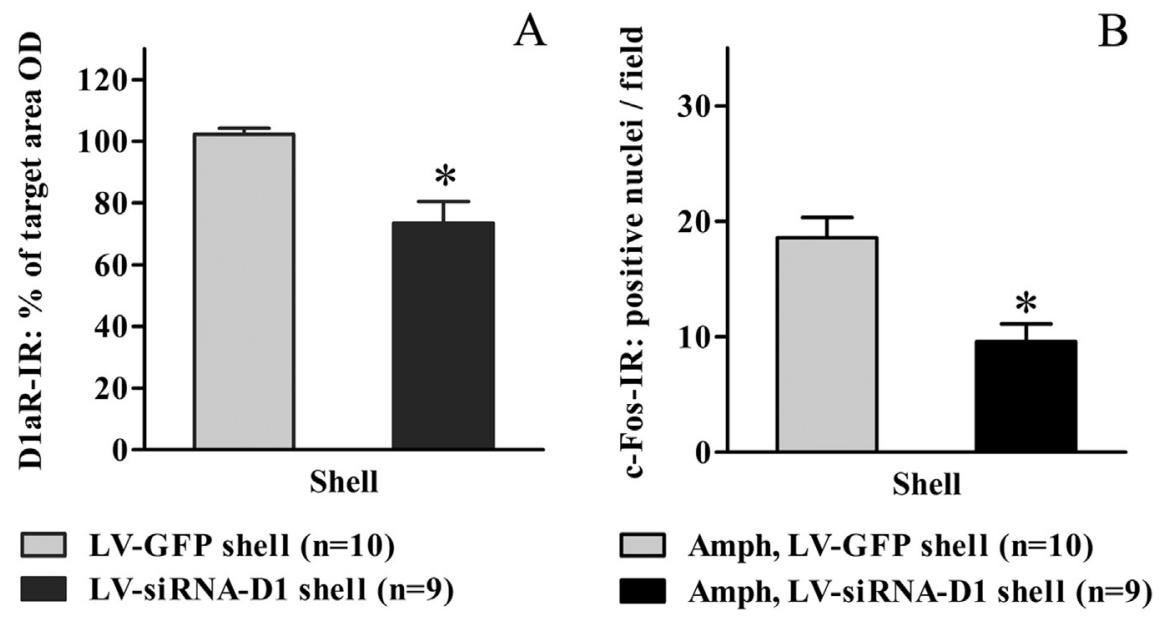

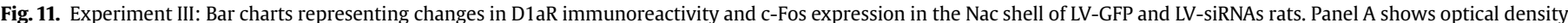

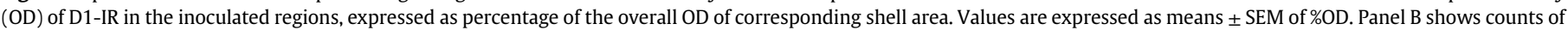

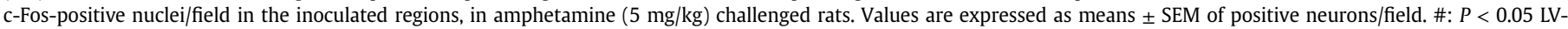
siRNAs vs LV-GFP groups, One -way ANOVA.

brain, with consequent loss of site specificity. A second advantage of LV-RNAi is that the distribution and degree of functional impairment can be precisely determined, while this is unfeasible in the case of competitive receptor ligands. Therefore, by using LVsiRNA for D1aR, the present study provides experimental evidence that DA D1aRs in the NAc shell rather than those in the core are essential for cocaine reinforcement.

\section{Authors' contribution}

GDC was responsible for the study concept and design. AB and JLD designed, prepared and validated lentiviral vectors. DL, VV, FC and GP performed surgeries for catheter implant and vector inoculation. DL, FC and AS performed SA experiments. AP performed immunohistochemical stainings and analysis. DL and AP performed statistical analysis and drafted the manuscript. CC and GDC provided critical review for important intellectual content. All authors critically reviewed content and approved final version for publication.

\section{Acknowledgments}

This study was supported by funds from Dipartimento Politiche Antidroga, Presidenza del Consiglio dei Ministri (DPA 0000757 p2.64.4.19 and DPA 0001151 p-4.35.14); Regione Autonoma della Sardegna (RAS) (n.7249/1118) and Fondazione Banco di Sardegna (n.838/2011.218). The authors acknowledge the National Institute on Drug Abuse (NIDA, Research Triangle Park, NC, USA) for supply of heroin. The authors acknowledge Dr. Annesha Sil for her contribution in editing of the language.

\section{References}

Alderson, H.L., Parkinson, J.A., Robbins, T.W., Everitt, B.J., 2001. The effects of excitotoxic lesions of the nucleus accumbens core or shell regions on intravenous heroin self-administration in rats. Psychopharmacol. Berl. 153, 455-463.

Aragona, B.J., Cleaveland, N.A., Stuber, G.D., Day, J.J., Carelli, R.M., Wightman, R.M., 2008. Preferential enhancement of dopamine transmission within the nucleus accumbens shell by cocaine is attributable to a direct increase in phasic dopamine release events. J. Neurosci. 28, 8821-8831. 
Bachtell, R.K., Whisler, K., Karanian, D., Self, D.W., 2005. Effects of intra-nucleus accumbens shell administration of dopamine agonists and antagonists on cocaine-taking and cocaine-seeking behaviors in the rat. Psychopharmacol. Berl. 183, 41-53.

Bahi, A., 2013. Viral-mediated knockdown of mGluR7 in the nucleus accumbens mediates excessive alcohol drinking and increased ethanol-elicited conditioned place preference in rats. Neuropsychopharmacology 38, 2109-2119.

Bahi, A., Dreyer, J.L., 2012. Involvement of nucleus accumbens dopamine D1 receptors in ethanol drinking, ethanol-induced conditioned place preference, and ethanol-induced psychomotor sensitization in mice. Psychopharmacol. Berl. 222, 141-153.

Bahi, A., Dreyer, J.L., 2013. Striatal modulation of BDNF expression using microRNA124a-expressing lentiviral vectors impairs ethanol-induced conditioned-place preference and voluntary alcohol consumption. Eur. J. Neurosci. 38, 2328-2337.

Bahi, A., Boyer, F., Kafri, T., Dreyer, J.L., 2004. CD81-induced behavioural changes during chronic cocaine administration: in vivo gene delivery with regulatable lentivirus. Eur. J. Neurosci. 19, 1621-1633.

Bahi, A., Boyer, F., Kafri, T., Dreyer, J.L., 2005. In vivo gene silencing of CD81 by lentiviral expression of small interference RNAs suppresses cocaine-induced behaviour. J. Neurochem. 92, 1243-1255.

Bari, A.A., Pierce, R.C., 2005. D1-like and D2 dopamine receptor antagonists administered into the shell subregion of the rat nucleus accumbens decrease cocaine, but not food, reinforcement. Neuroscience 135, 959-968.

Brenhouse, H.C., Stellar, J.R., 2006. c-Fos and deltaFosB expression are differentially altered in distinct subregions of the nucleus accumbens shell in cocainesensitized rats. Neuroscience 137, 773-80. Epub 2005 Dec 5.

Caine, S.B., Heinrichs, S.C., Coffin, V.L., Koob, G.F., 1995. Effects of the dopamine D1antagonist SCH 23390 microinjected into the accumbens, amygdala or striatum on cocaine self-administration in the rat. Brain Res. 692, 47-56.

Carboni, E., Imperato, A., Perezzani, L., Di Chiara, G., 1989. Amphetamine, cocaine, phencyclidine and nomifensine increase extracellular dopamine concentrations preferentially in the nucleus accumbens of freely moving rats. Neuroscience 28 , 653-661.

Corbit, L.H., Balleine, B.W., 2011. The general and outcome-specific forms of Pavlovian-instrumental transfer are differentially mediated by the nucleus accumbens core and shell. J. Neurosci. 31, 11786-11794.

Corbit, L.H., Muir, J.L., Balleine, B.W., 2001. The role of the nucleus accumbens in instrumental conditioning: evidence of a functional dissociation between accumbens core and shell. J. Neurosci. 21, 3251-3260.

Di Chiara, G., 2002. Nucleus accumbens shell and core dopamine: differential role in behavior and addiction. Behav. Brain. Res. 137, 75-114.

Di Chiara, G., Imperato, A., 1988. Drugs abused by humans preferentially increase synaptic dopamine concentrations in the mesolimbic system of freely moving rats. Proc. Natl. Acad. Sci. U. S. A. 85, 5274-5278.

Di Chiara, G., Bassareo, V., Fenu, S., De Luca, M.A., Spina, L., Cadoni, C., et al., 2004 Dopamine and drug addiction: the nucleus accumbens shell connection. Neuropharmacology 47, 227-241.

Di Ciano, P., 2008. Distinct contributions of dopamine receptors in the nucleus accumbens core or shell to established cocaine reinforcement under a secondorder schedule. Eur. Neuropsychopharmacol. 18, 888-896.

Di Ciano, P., Coury, A., Depoortere, R.Y., Egilmez, Y., Lane, J.D., EmmettOglesby, M.W., et al., 1995. Comparison of changes in extracellular dopamine concentrations in the nucleus accumbens during intravenous selfadministration of cocaine or d-amphetamine. Behav. Pharmacol. 6, 311-322.

Drevets, W.C., Price, J.C., Kupfer, D.J., Kinahan, P.E., Lopresti, B., Holt, D., et al., 1999. PET measures of amphetamine-induced dopamine release in ventral versus dorsal striatum. Neuropsychopharmacology 21, 694-709.

Drevets, W.C., Gautier, C., Price, J.C., Kupfer, D.J., Kinahan, P.E., Grace, A.A., et al., 2001. Amphetamine-induced dopamine release in human ventral striatum correlates with euphoria. Biol. Psychiatry 49, 81-96.

Ettenberg, A., Pettit, H.O., Bloom, F.E., Koob, G.F., 1982. Heroin and cocaine intravenous self-administration in rats: mediation by separate neural systems. Psychopharmacol. Berl. 78, 204-209.

Fire, A., Xu, S., Montgomery, M.K., Kostas, S.A., Driver, S.E., Mello, C.C., 1998. Potent and specific genetic interference by double-stranded RNA in Caenorhabditis elegans. Nature 391, 806-811.

Goeders, N.E., Smith, J.E., 1983. Cortical dopaminergic involvement in cocaine reinforcement. Science 221, 773-775.

Graybiel, A.M., Moratalla, R., Robertson, H.A., 1990. Amphetamine and cocaine induce drug-specific activation of the c-fos gene in striosome-matrix compartments and limbic subdivisions of the striatum. Proc. Natl. Acad. Sci. U. S. A. 87, 6912-6916.

Groenewegen, H.J., Wright, C.I., Beijer, A.V., Voorn, P., 1999. Convergence and segregation of ventral striatal inputs and outputs. Ann. N. Y. Acad. Sci. 877, 49-63.

Hamilton, A.J., Baulcombe, D.C., 1999. A species of small antisense RNA in posttranscriptional gene silencing in plants. Science 286, 950-952.

Hannon, G.J., 2002. RNA interference. Nature 418, 244-251.

Hannon, G.J., Rossi, J.J., 2004. Unlocking the potential of the human genome with RNA interference. Nature 431, 371-378.
Heimer, L., Alheid, G.F., de Olmos, J.S., Groenewegen, H.J., Haber, S.N., Harlan, R.E., et al., 1997. The accumbens: beyond the core-shell dichotomy. J. Neuropsychiatry Clin. Neurosci. 9, 354-381.

Ikemoto, S., 2003. Involvement of the olfactory tubercle in cocaine reward: intracranial self-administration studies. J. Neurosci. 23, 9305-9311.

Jensen, S., Gassama, M.P., Heidmann, T., 1999. Cosuppression of I transposon activity in Drosophila by I-containing sense and antisense transgenes. Genetics 153, 1767-1774.

Koob, G.F., Le, H.T., Creese, I., 1987. The D1 dopamine receptor antagonist SCH 23390 increases cocaine self-administration in the rat. Neurosci. Lett. 79, 315-320.

Lecca, D., Cacciapaglia, F., Valentini, V., Acquas, E., Di Chiara, G., 2007a. Differential neurochemical and behavioral adaptation to cocaine after response contingent and noncontingent exposure in the rat. Psychopharmacol. Berl. 191, 653-667.

Lecca, D., Valentini, V., Cacciapaglia, F., Acquas, E., Di Chiara, G., 2007b. Reciprocal effects of response contingent and noncontingent intravenous heroin on in vivo nucleus accumbens shell versus core dopamine in the rat: a repeated sampling microdialysis study. Psychopharmacol. Berl. 194, 103-116.

Levey, A.I., Hersch, S.M., Rye, D.B., Sunahara, R.K., Niznik, H.B., Kitt, C.A., Price, D.L., Maggio, R., Brann, M.R., Ciliax, B.J., 1993. Localization of D1 and D2 dopamine receptors in brain with subtype-specific antibodies. Proc. Natl. Acad. Sci. U. S. A. $90,8861-8865$.

Leyton, M., Boileau, I., Benkelfat, C., Diksic, M., Baker, G., Dagher, A., 2002. Amphetamine-induced increases in extracellular dopamine, drug wanting, and novelty seeking: a PET/[11C]raclopride study in healthy men. Neuropsychopharmacology 27, 1027-1035.

Luedtke, R.R., Griffin, S.A., Conroy, S.S., Jin, X., Pinto, A., Sesack, S.R., 1999. Immunoblot and immunohistochemical comparison of murine monoclonal antibodies specific for the rat D1a and D1b dopamine receptor subtypes. J. Neuroimmunol. 101, 170-187.

Maldonado, R., Robledo, P., Chover, A.J., Caine, S.B., Koob, G.F., 1993. D1 dopamine receptors in the nucleus accumbens modulate cocaine self-administration in the rat. Pharmacol. Biochem. Behav. 45, 239-242.

Martinez, D., Slifstein, M., Broft, A., Mawlawi, O., Hwang, D.R., Huang, Y., et al., 2003. Imaging human mesolimbic dopamine transmission with positron emission tomography. Part II: amphetamine-induced dopamine release in the functional subdivisions of the striatum. J. Cereb. Blood Flow. Metab. 23, 285-300.

Moratalla, R., Xu, M., Tonegawa, S., Graybiel, A.M., 1996. Cellular responses to psychomotor stimulant and neuroleptic drugs are abnormal in mice lacking the D1 dopamine receptor. Proc. Natl. Acad. Sci. U. S. A. 93, 14928-14933.

Muly, E.C., Maddox, M., Khan, Z.U., 2010. Distribution of D1 and D5 dopamine receptors in the primate nucleus accumbens. Neuroscience 169, 1557-1566.

Ortiz, O., Delgado-García, J.M., Espadas, I., Bahí, A., Trullas, R., Dreyer, J.L., et al., 2010. Associative learning and CA3-CA1 synaptic plasticity are impaired in D1R null, Drd1a-/- mice and in hippocampal siRNA silenced Drd1a mice. J. Neurosci. 30, $12288-12300$.

Parkinson, J.A., Dalley, J.W., Cardinal, R.N., Bamford, A., Fehnert, B., Lachenal, G., et al., 2002. Nucleus accumbens dopamine depletion impairs both acquisition and performance of appetitive Pavlovian approach behaviour: implications for mesoaccumbens dopamine function. Behav. Brain Res. 137, 149-163.

Pettit, HO., Justice Jr., J.B., 1989. Dopamine in the nucleus accumbens during cocaine self-administration as studied by in vivo microdialysis. Pharmacol. Biochem. Behav. 34, 899-904.

Pettit, H.O., Justice Jr., J.B., 1991. Effect of dose on cocaine self-administration behavior and dopamine levels in the nucleus accumbens. Brain Res. 539, 94-102.

Pettit, H.O., Ettenberg, A., Bloom, F.E., Koob, G.F., 1984. Destruction of dopamine in the nucleus accumbens selectively attenuates cocaine but not heroin selfadministration in rats. Psychopharmacol. Berl. 84, 167-173.

Pontieri, F.E., Tanda, G., Di Chiara, G., 1995. Intravenous cocaine, morphine, and amphetamine preferentially increase extracellular dopamine in the "shell" as compared with the "core" of the rat nucleus accumbens. Proc. Natl. Acad. Sci. U. S. A. 92, 12304-12308.

Roberts, D.C., Koob, G.F., Klonoff, P., Fibiger, H.C., 1980. Extinction and recovery of cocaine self-administration following 6-hydroxydopamine lesions of the nucleus accumbens. Pharmacol. Biochem. Behav. 12, 781-787.

Rodd-Henricks, Z.A., McKinzie, D.L., Li, T.K., Murphy, J.M., McBride, W.J., 2002 Cocaine is self-administered into the shell but not the core of the nucleus accumbens of Wistar rats. J. Pharmacol. Exp. Ther. 303, 1216-1226.

Scherr, M., Battmer, K., Ganser, A., Eder, M., 2003. Modulation of gene expression by lentiviral-mediated delivery of small interfering RNA. Cell Cycle 2, 251-257.

Sharp, P.A., Zamore, P.D., 2000. Molecular biology. RNA interference. Science 287, $2431-2433$.

Van den Haute, C., Eggermont, K., Nuttin, B., Debyser, Z., Baekelandt, V., 2003. Lentiviral vector-mediated delivery of short hairpin RNA results in persistent knockdown of gene expression in mouse brain. Hum. Gene Ther. 14, 1799-1807.

Veeneman, M.M., Broekhoven, M.H., Damsteegt, R., Vanderschuren, L.J., 2012. Distinct contributions of dopamine in the dorsolateral striatum and nucleus accumbens shell to the reinforcing properties of cocaine. Neuropsychopharmacology 37, 487-498.

Wise, R.A., Newton, P., Leeb, K., Burnette, B., Pocock, D., Justice Jr., J.B., 1995. Fluctuations in nucleus accumbens dopamine concentration during intravenous cocaine self-administration in rats. Psychopharmacol. Berl. 120, 10-20. 